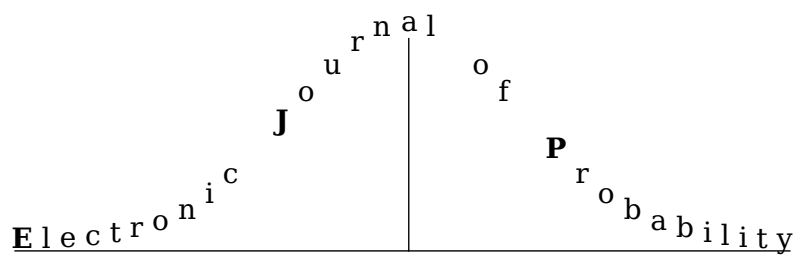

Electron. J. Probab. 25 (2020), article no. 95, 1-33.

ISSN: 1083-6489 https://doi.org/10.1214/20-EJP494

\title{
On uniqueness of solutions to martingale problems - counterexamples and sufficient criteria
}

\author{
Jan Kallsen* $\quad$ Paul Krühner ${ }^{\dagger}$
}

\begin{abstract}
The dynamics of a Markov process are often specified by its infinitesimal generator or, equivalently, its symbol. This paper contains examples of analytic symbols which do not determine the law of the corresponding Markov process uniquely. These examples also show that the law of a polynomial process in the sense of $[4,5,11]$ is not necessarily determined by its generator if it has jumps. On the other hand, we show that a combination of smoothness of the symbol and ellipticity warrants uniqueness in law. The proof of this result is based on proving stability of univariate marginals relative to some properly chosen distance.
\end{abstract}

Keywords: symbol; martingale problem; uniqueness; polynomial process; Markov process; pseudo-differential operator; jump processes.

MSC2020 subject classifications: 47G30; 60J35; 60J75.

Submitted to EJP on May 8, 2019, final version accepted on July 4, 2020.

Supersedes arXiv: $1607.02998 \mathrm{v} 1$.

\section{Introduction}

Consider a system whose state at time $t$ is represented by a vector $X(t)$ in $\mathbb{R}^{d}$. In applications the dynamics of such a system are often described by specifying how $X(t)$ changes as a function of the current state $X(t)$. In a deterministic setup this is typically expressed in terms of an ordinary differential equation. If, on the other hand, $X(t)$ is random, it may be viewed as a Markov process whose local dynamics can be specified in terms of a stochastic differential equation, its infinitesimal generator, its local semimartingale characteristics, or its symbol. As in the deterministic case, this immediately leads to the question of existence and uniqueness of a stochastic process exhibiting the given local dynamics.

This can be rephrased in terms of existence and uniqueness of the solution to a corresponding martingale problem. Existence is known to hold under relatively weak continuity conditions, see, for example, [9, Theorem 4.5.4], [16, Theorem 3.15], [3,

${ }^{*}$ Kiel University, Germany. E-mail: kallsen@math.uni-kiel. de

${ }^{\dagger}$ University of Liverpool, United Kingdom. E-mail: P. eisenberg@liverpool .ac.uk 
Theorem 3.24], [23, Corollary 3.2], [24, Theorem 1.1], [8, Theorem 5.36], and Theorem 2.7 below. The solution can often be chosen as a strong Markov process, see once more [24, Theorem 1.1] and Theorem 2.7, which both rely on the general sufficient conditions in [9, Theorems 4.5.11(b) and 4.5.19].

For continuous processes uniqueness holds for Lipschitz- resp. Hölder-continuous coefficients or under some ellipticity condition, cf. e.g. [27]. The situation is less obvious for processes with jumps, which are the main focus of this paper. Lipschitz conditions only help for generators which have a natural representation as an SDE, which often is not the case. Ellipticity, on the other hand, requires a continuous martingale part to be present, which often is not the case either.

This piece of research is motivated by the desire to come up with a general uniqueness result for Markov processes that may not have a continuous martingale part or a natural representation as a SDE. In this context we share the point of view of $[16,20,3]$ that it is natural to study Markov processes through their symbol. Indeed, e.g. weak convergence of a sequence of Levy processes corresponds to pointwise convergence of their symbols.

From the analogy to ODE's one may expect uniqueness to hold if the symbol of the process depends smoothly on the state $X(t)$. Unfortunately, smoothness alone does not seem to suffice in order to warrant uniqueness. In Section 3 we present two examples of even analytic symbols where uniqueness in law of the corresponding Markov process does not hold. This is the first main result of this paper. These examples also show that the law of a polynomial process in the sense of $[4,5,11]$ is not in general uniquely determined by its generator. This complements a positive result by [10, Theorem 5.3], which states that uniqueness does in fact hold for univariate continuous polynomial processes.

Section 4 contains a positive result, which is the second main contribution of this paper. It is shown that the combination of sufficient smoothness and ellipticity warrants uniqueness in law. In contrast to [30, Theorem 4.3] and related results, the continuous martingale part may vanish. The probably closest relative to our Theorem 4.4 below is [2, Theorem 2.8] which also relies on smoothness and ellipticity of the symbol. However, Böttcher requires a certain boundedness for derivatives of any order while we need this condition only for finitely many derivatives. Nevertheless, [2, Theorem 2.8] is not a special case of our Theorem 4.4 below. Another closely related result is [16, Theorem 5.24] which, however, requires the symbol to be real.

Uniqueness results have been obtained by a number of different approaches, cf. [20] for an overview. From a very rough perspective, the most commonly used techniques are

- SDE methods where uniqueness is often obtained from fixed-point arguments,

- construction of a solution to the backward equation, i.e. construction of solutions for the associated abstract Cauchy problem, and

- so-called interlacing techniques which allow to add finitely many jumps.

By contrast, our approach is based on establishing stability of the univariate marginals relative to a properly chosen distance. This kind of reasoning seems to be new and it constitutes the third main contribution of this paper.

The paper is structured as follows. In Section 2 we recall various notions and properties concerning symbols and martingale problems. Moreover, we prove the existence of a Markovian solution under continuity conditions, making use of general results in [9, Chapter 4]. Subsequently, we present examples showing that smoothness of the symbol does not imply uniqueness of the solution to a martingale problem. In Section 4 a uniqueness result under smoothness and some mild ellipticity of the symbol is stated. Section 5 contains proofs. In the appendix, we recall some facts on complex measures. 
On unique solutions to martingale problems

\subsection{Notation}

$d \in \mathbb{N}$ generally denotes the dimension of the space under consideration. We denote the trace of a matrix $C \in \mathbb{R}^{d \times d}$ by $\operatorname{Tr}(C)$. For any two vectors $x, y \in \mathbb{C}^{d}$ we define the standard bilinear form $x y:=\sum_{j=1}^{d} x_{j} y_{j}$. Moreover, we set $C x:=\left(\sum_{k=1}^{d} C_{j k} x_{k}\right)_{j=1, \ldots, d}$ and $y C x:=y(C x)$ for any matrix $C \in \mathbb{C}^{d \times d}$ and vectors $x, y \in \mathbb{C}^{d}$. The set of positive semidefinite $d \times d$-matrices is denoted by $S^{d}$. We fix a truncation function $\chi: \mathbb{R}^{d} \rightarrow \mathbb{R}^{d}$, i.e. $\chi$ is measurable, bounded and it equals the identity in a neighbourhood of zero. W.l.o.g. we suppose that $\chi(x)=x$ for $|x| \leq 1$, where $|x|:=\left(\sum_{j=1}^{d}\left|x_{j}\right|^{2}\right)^{1 / 2}$ denotes the Euclidean norm on $\mathbb{R}^{d}$. We write $B(x, r):=\left\{y \in \mathbb{R}^{d}:|x-y|<r\right\}$ for the open ball with radius $r>0$ centered at $x \in \mathbb{R}^{d}$. We denote the gradient of $f \in C^{1}\left(\mathbb{R}^{d}, \mathbb{C}\right)$ by $\nabla f(x):=\left(\partial_{1} f, \ldots, \partial_{d} f\right)$, $x \in \mathbb{R}^{d}$, the Hessian of $f \in C^{2}\left(\mathbb{R}^{d}, \mathbb{C}\right)$ by $H f(x):=\left(\partial_{j k}^{2} f(x)\right)_{j, k=1}^{d}, x \in \mathbb{R}^{d}$, and the Laplacian of $f \in C^{2}\left(\mathbb{R}^{d} \times \mathbb{R}^{d}, \mathbb{C}\right)$ by $\Delta f(x):=\sum_{j=1}^{d} \partial_{j}^{2} f(x), x \in \mathbb{R}^{d}$. For functions $f: \mathbb{R}^{d} \times \mathbb{R}^{d} \rightarrow \mathbb{C}$ we write $\nabla_{1} f(x, y):=(\nabla f(\cdot, y))(x), x, y \in \mathbb{R}^{d}$ if $f$ is differentiable with respect to the first coordinate and $\Delta_{1} f(x, y):=(\Delta f(\cdot, y))(x), x, y \in \mathbb{R}^{d}$ for sufficiently smooth $f$. If $f$ is smooth enough in the second coordinate, $\nabla_{2} f(x, y)$ and $H_{2} f(x, y)$ are defined accordingly. By $\hat{C}\left(\mathbb{R}^{d}\right)$ (resp. $\hat{C}\left(\mathbb{R}^{d}, \mathbb{C}\right)$ ) we denote the set of real-valued (resp. complex-valued) continuous functions on $\mathbb{R}^{d}$ that vanish in $\infty$. Similarly, $\bar{C}\left(\mathbb{R}^{d}\right)$ stands for the set of bounded continuous functions on $\mathbb{R}^{d}$. The Skorokhod space of càdlàg functions is denoted as $\mathbb{D}:=D_{\mathbb{R}^{d}}[0, \infty):=\left\{\alpha: \mathbb{R}_{+} \rightarrow \mathbb{R}^{d}: \alpha\right.$ càdlàg $\}$. The greatest integer less or equal $x \in \mathbb{R}$ is written as $[x]$. Further unexplained notation is used as in $[9,21]$.

\section{The symbol and the existence theorem}

We start by recalling the notion of the symbol and its associated martingale problem, cf. [20, 3]. A systematic theory for symbols was first developed by Hoh [14, 15, 17]. Other important references include [18], which is more in view of strongly continuous semigroups, and [1], who developed a theory for symbols on nuclear separable spaces.

Definition 2.1. 1. A measurable function $q: \mathbb{R}^{d} \times \mathbb{R}^{d} \rightarrow \mathbb{C}$ is a symbol if $q(x, \cdot)$ is a Lévy exponent for all $x \in \mathbb{R}^{d}$, i.e. there are functions $b: \mathbb{R}^{d} \rightarrow \mathbb{R}^{d}, c: \mathbb{R}^{d} \rightarrow S^{d}$, and $F: \mathbb{R}^{d} \times \mathcal{B}\left(\mathbb{R}^{d}\right) \rightarrow \mathbb{R}^{d}$ such that $F(x, \cdot)$ is a Lévy measure and

$$
q(x, u)=i u b(x)-\frac{1}{2} u c(x) u+\int\left(e^{i u y}-1-i u \chi(y)\right) F(x, d y)
$$

for any $x, u \in \mathbb{R}^{d}$. We call a symbol $q: \mathbb{R}^{d} \times \mathbb{R}^{d} \rightarrow \mathbb{C}(f$-)Hölder continuous if there is a continuous bounded function $f: \mathbb{R}^{d} \rightarrow \mathbb{R}_{+}$such that

$$
|q(x, u)-q(y, u)| \leq f(x-y)\left(1+|u|^{2}\right), \quad x, y, u \in \mathbb{R}^{d} .
$$

2. If $q$ denotes a symbol, an adapted càdlàg $\mathbb{R}^{d}$-valued stochastic process $X$ on some filtered probability space $\left(\Omega, \mathscr{F},\left(\mathscr{F}_{t}\right)_{t \geq 0}, P\right)$ is called solution to the $q$-martingale problem if the process

$$
M_{u}(t):=\exp (i u X(t))-\int_{0}^{t} q(X(s), u) \exp (i u X(s)) d s
$$

is a local martingale for any $u \in \mathbb{R}^{d}$. Uniqueness for the $q$-martingale problem means that any two solutions $X, Y$ to the $q$-martingale problem with the same initial law (i.e. $X(0)$ has the same law as $Y(0)$ ) have the same distribution. Finally, we say that existence holds for the $q$-martingale problem if, for any probability measure $\mu$, there is a solution $X$ to the $q$-martingale problem with initial law $P^{X(0)}=\mu$. 
Remark 2.2. The functions $b, c$ in the Lévy-Khintchine representation (2.1) are measurable and $F$ is a transition kernel from $\mathbb{R}^{d}$ to $\mathbb{R}^{d}$, which can e.g. be derived from the construction in [21, Lemma II.2.44]. As opposed to $c$ and $F$, the drift coefficient $b$ depends on the choice of $\chi$, cf. [29, Theorem 8.1]. We call $(b(x), c(x), F(x, \cdot))$ Lévy-Khintchine triplet of the Lévy exponent $q(x, \cdot)$.

For further use we make the following observation.

Remark 2.3. Let $q$ be a symbol. A simple Taylor approximation argument shows that for any $x \in \mathbb{R}^{d}$ there exists $C_{x}<\infty$ such that $|q(x, u)| \leq C_{x}\left(1+|u|^{2}\right), u \in \mathbb{R}^{d}$. Hence

$$
\int|f(u) q(x, u)| d u<\infty
$$

for any Schwartz function $f$ in the sense of [12, Definition 2.2.1] and any $x \in \mathbb{R}^{d}$.

In order to relate a symbol to a martingale problem in the sense of [9, Section 4.3], we define an operator corresponding to the symbol.

Definition 2.4. Let $q$ be a symbol. The operator $\mathcal{A}$ associated with $q$ is defined as

$$
\mathcal{A} f(x):=\int q(x, u) \check{f}(u) e^{i u x} d u
$$

where $x \in \mathbb{R}^{d}, f$ is any real-valued Schwartz function, and

$$
\check{f}: \mathbb{R}^{d} \rightarrow \mathbb{C}, \quad u \mapsto \frac{1}{(2 \pi)^{d}} \int f(x) e^{-i u x} d x,
$$

denotes the inverse Fourier transform of $f$.

This operator can be expressed in terms of the Lévy-Khintchine triplet.

Lemma 2.5. Let $q$ be a symbol, $\mathcal{A}$ the operator associated with $q$, and $(b, c, F)$ the triplet of $q$. Then

$$
\mathcal{A} f(x)=\nabla f(x) b(x)+\frac{1}{2} \operatorname{Tr}(H f(x) c(x))+\int(f(x+y)-f(x)-\nabla f(x) \chi(y)) F(x, d y)
$$

for any real-valued Schwartz function $f$ and any $x \in \mathbb{R}^{d}$.

Proof. This follows from [12, Proposition 2.3.22].

Let $q$ be a symbol with associated operator $\mathcal{A}$. Moreover, denote by $\mathcal{B}$ the restriction of $\mathcal{A}$ to the set of real-valued Schwartz functions $f$ such that $\mathcal{A} f$ is bounded. The following lemma shows that any solution $X$ to the $q$-martingale problem is a solution to the martingale problem in the sense of [9, Section 4.3] for $\mathcal{B}$. Under suitable conditions the converse is also true, cf. Theorem 2.7(2) below.

Lemma 2.6. Let $X$ be a solution to the $q$-martingale problem, $f$ a real-valued Schwartz function such that $\mathcal{A} f$ is bounded, and

$$
M_{f}(t):=f(X(t))-\int_{0}^{t} \mathcal{A} f(X(s)) d s
$$

for any $t \geq 0$. Then $M_{f}$ is a martingale.

Proof. Proposition 5.1 states that $X$ is a semimartingale. Furthermore, its local characteristics is given by $\left(b\left(X_{-}\right), c\left(X_{-}\right), F\left(X_{-}, \cdot\right)\right)$. Thus Itō's formula for the local characteris- 
On unique solutions to martingale problems

tics [22, Proposition 2.5] together with Lemma 2.5 yield that

$$
\begin{aligned}
G(t, A) & :=\int 1_{A}\left(f\left(X_{t-}+x\right)-f\left(X_{t-}\right)\right) F_{t}(d x), \quad A \in \mathcal{B} \text { with } 0 \notin A, \\
\gamma(t) & :=\nabla f(X(t-)) c(X(t-)) \nabla f(X(t-)), \\
\beta(t) & :=\mathcal{A} f(X(t-))+\int(h(y)-y) G(t, d y),
\end{aligned}
$$

is a version of the local characteristics of $f(X)$ relative to the truncation function $h$. By [21, Theorem II.2.42]

$$
f(X(t))-\int_{0}^{t} \mathcal{A} f(X(s)) d s
$$

is a local martingale. However, $M_{f}$ is bounded on compact time intervals and hence it is a martingale.

We now state an essentially well-known existence result which follows from [9, Theorems 4.5.4, 4.5.11, 4.5.19]. The boundedness assumption on the function $g$ in the following theorem amounts to saying that $b$ and $c$ are bounded and that $F$ has bounded second moment.

Theorem 2.7 (Existence). Let $q$ be a continuous symbol with associated operator $\mathcal{A}$ and triplet $(b, c, F)$. Assume that

$$
g: \mathbb{R}^{d} \rightarrow \mathbb{R} \cup\{\infty\}, \quad x \mapsto|b(x)|+\operatorname{Tr}(c(x))+\int|y|^{2} F(x, d y)
$$

is bounded by some finite constant. Then the following statements hold.

1. For any probability measure $\mu$ on $\mathbb{R}^{d}$ there is a solution $X$ to the $q$-martingale problem with $P^{X(0)}=\mu$.

2. A stochastic process $X$ is a solution to the $q$-martingale problem if and only if

$$
M_{f}(t):=f(X(t))-\int_{0}^{t} \mathcal{A} f(X(s)) d s, \quad t \in \mathbb{R}_{+}
$$

defines a martingale for any real-valued Schwartz function $f$ (or, equivalently, any smooth function with compact support), i.e. if and only if $X$ is a solution to the martingale problem $\mathcal{A}$ in the sense of [9, Section 4.3].

3. The operator $\mathcal{A}$ has the following properties:

(a) its range is contained in $\hat{C}\left(\mathbb{R}^{d}\right)$,

(b) it satisfies the positive maximum principle in the sense of [9, p. 165], i.e.

$$
0 \leq f\left(x_{0}\right)=\sup _{x \in \mathbb{R}^{d}} f(x)
$$

implies $\mathcal{A} f\left(x_{0}\right) \leq 0$ for any real-valued Schwartz function $f$ and any $x_{0} \in \mathbb{R}^{d}$, and

(c) it is conservative, i.e. there is a bounded sequence of real-valued Schwartz functions $\left(f_{n}\right)_{n \in \mathbb{N}}$ which converges pointwise to 1 such that $\left(\mathcal{A} f_{n}\right)_{n \in \mathbb{N}}$ is a bounded sequence which converges pointwise to 0 .

4. It is possible to choose measures $\left(P_{x}\right)_{x \in \mathbb{R}^{d}}$ on the Skorokhod space such that the canonical process $X$ is a solution to the $q$-martingale problem with $X(0)=x$ a.s. under $P_{x}, x \in \mathbb{R}^{d}$ and such that $x \mapsto P_{x}(X(t) \in A)$ is measurable for any $t \geq 0$ and any Borel set $A \subset \mathbb{R}^{d}$. Moreover, $\left(X,\left(P_{x}\right)_{x \in \mathbb{R}^{d}}\right)$ is strong Markov. 
5. If the $q$-martingale problem has several solutions for some initial law $P^{X(0)}=\mu$, then there are several families of measures $\left(P_{x}\right)_{x \in \mathbb{R}^{d}}$ as in (4).

6. Finally,

$$
q(x, u)=\lim _{t \downarrow 0} \frac{E_{x}\left(e^{i u(X(t)-x)}\right)-1}{t}, \quad x, u \in \mathbb{R}^{d},
$$

where $E_{x}$ denotes expectation relative to $P_{x}$.

The proof is to be found in Section 5.1.

Remark 2.8. 1. Provided that uniqueness holds, the boundedness condition in Theorem 2.7 can be relaxed by localisation, i.e. by applying [9, Theorems 4.6.2 or 4.6.3]. Without assuming uniqueness, the existence of a Markovian solution has recently and independently been shown in [24, Theorem 1.1]. Instead of boundedness of $g$ in Theorem 2.7 Kühn requires

$$
\begin{aligned}
\sup _{|x| \leq R} \sup _{|u| \leq 1}|q(x, u)|<\infty, \quad R \in \mathbb{R}_{+}, \\
\lim _{R \rightarrow \infty} \sup _{|x| \leq R} \sup _{|u| \leq 1 / R}|q(x, u)|=0 .
\end{aligned}
$$

A straightforward calculation shows that boundedness of $g$ actually implies (2.2, 2.3).

2. For his related existence result [16, Theorem 3.15] (cf. also [3, Theorem 3.24]), Hoh requires slightly weaker conditions. However, he focuses on statement (1), i.e., the existence of a Markovian solution is not considered. The same is true for [8, Theorem 5.36].

3. Statement (6) of Theorem 2.7 means that $-q$ is a symbol in the sense of [20].

The assumption on the triplet in Theorem 2.7 can be replaced by a smoothness condition on the symbol:

Corollary 2.9. Let $q$ be a continuous symbol such that $u \mapsto q(x, u)$ is twice differentiable with bounded gradient $x \mapsto \nabla_{2} q(x, 0)$ and bounded Hessian $x \mapsto H_{2} q(x, 0)$. Then statements (1-6) in Theorem 2.7 hold.

Proof. W.l.o.g. $|\chi(y)|=0$ for $|y|>1$. Fix $x \in \mathbb{R}^{d}$ and define the finite measure $F_{x}(A):=$ $F(x, A \backslash \overline{B(0,1)}), A \in \mathcal{B}\left(\mathbb{R}^{d}\right)$ as well as $\bar{F}_{x}:=F(x, \cdot)-F_{x}$. We denote the Fourier transform of $F_{x}$ by

$$
\hat{F}_{x}(u):=\int e^{i u y} F_{x}(d y), \quad u \in \mathbb{R}^{d} .
$$

Observe that

$$
\hat{F}_{x}(u)=q(x, u)-i u b(x)+\frac{1}{2} u c(x) u-\int\left(e^{i u y}-1-i u y\right) \bar{F}_{x}(d y)+\hat{F}_{x}(0) .
$$

Dominated convergence and $\int|y|^{2} \bar{F}_{x}(d y)<\infty$ yield that $\hat{F}_{x}$ is twice differentiable in 0 . By [7, Lemma A.1], this implies that $F_{x}$ has finite second moments given by $\int y_{j}^{2} F_{x}(d y)=-\hat{F}_{x}^{\prime \prime}(0), j=1, \ldots, d$. Again by dominated convergence we obtain

$$
\begin{aligned}
\left(H_{2} q(x, 0)\right)_{j j} & =-c(x)_{j j}-\int y_{j}^{2} \bar{F}_{x}(d y)-\int y_{j}^{2} F_{x}(d y) \\
& =-c(x)_{j j}-\int y_{j}^{2} F(x, d y), \quad j=1, \ldots, d .
\end{aligned}
$$

Boundedness of $H_{2} q(\cdot, 0)$ now yields that $\operatorname{Tr}(c(\cdot))$ and $\int|y|^{2} F(\cdot, d y)$ are bounded as well. 
Once more from dominated convergence we conclude

$$
\nabla_{2} q(x, 0)=i\left(b(x)+\int(y-\chi(y)) F(x, d y)\right) .
$$

Since $\int(y-\chi(y)) F(\cdot, d y)$ is a bounded function, $b$ is bounded as well. Theorem 2.7 now yields the assertion.

A remarkable result by van Casteren indicates that uniqueness almost implies the Feller property, cf. [25, Theorem 3.1] or [16, Proposition 5.18]. A missing growth condition along with a complete proof has been provided by Kühn [23, Theorem 1.1]. Applied to our context it reads as follows.

Proposition 2.10 (Feller property). Let $q$ be a symbol satisfying the requirements of Theorem 2.7. If uniqueness holds for the $q$-martingale problem, then there is a closed extension $\mathcal{C}$ of $\mathcal{A}$ which generates a strongly continuous positivity preserving contraction semigroup on $\hat{C}\left(\mathbb{R}^{d}\right)$. In other words, any solution to the martingale problem $\mathcal{A}$ is a Feller process.

Proof. Boundedness of $g$ in Theorem 2.7 implies that $\mathcal{A}$ maps Schwartz functions on a subset of $\hat{C}\left(\mathbb{R}^{d}\right)$. Since boundedness of $g$ also implies the growth conditions in [23, Lemma 3.1(i-iii)], the claim follows from [23, Theorem 1.1].

\section{Counterexamples}

In this section we provide an example of a real-valued analytic symbol which fails to have the uniqueness property in the sense of Definition 2.1. Moreover, we present a closely related example. Both correspond to polynomial processes in the sense of [4, 5], i.e. the extended operator $\mathcal{A}$ in the sense of [5, Definition 2.3] maps polynomials on polynomials of at most the same degree.

Example 3.1. There is an analytic symbol, namely

$$
q: \mathbb{R} \times \mathbb{R} \rightarrow \mathbb{R}, \quad(x, u) \mapsto \begin{cases}\frac{\cos (x u)-1}{x^{2}} & \text { for } x \neq 0, \\ -\frac{u^{2}}{2} & \text { otherwise },\end{cases}
$$

satisfying the requirements of Theorem 2.7 and having an entire extension to $\mathbb{C} \times \mathbb{C}$ where, however, uniqueness does not hold for the $q$-martingale problem.

Moreover, there are solutions $X, Y$ to the $q$-martingale problem with $X(0)=0=Y(0)$ and $P^{X(t)} \neq P^{Y(t)}$ for any $t>0$. More generally, there are strong Markov processes $X$, $Y$ on $\mathbb{R}^{d}$ with the above symbol which do not have the same law. Moreover, $X, Y$ are polynomial processes in the sense of [5, Definition 2.1] or [11, Definition 2.1]. Starting in $X(0)=0=Y(0)$, their $n$-th moment at time $t$ is given by

$$
E_{0}\left(X^{n}(t)\right)=E_{0}\left(Y^{n}(t)\right)=1_{\mathbb{N}}(n / 2) \frac{t^{n / 2}}{(n / 2) !} \prod_{k=1}^{n / 2}\left(2^{2 k-1}-1\right), \quad t \geq 0, \quad n \in \mathbb{N} .
$$

Proof. Suppose that the truncation function $\chi$ is continuous and anti-symmetric. Define $q$ as in (3.1). The function $q$ has an obvious entire extension. Define

$$
\begin{aligned}
b(x) & =0, \\
c(x) & =1_{\{x=0\}}, \\
F(x, \cdot) & :=1_{\{x \neq 0\}} \frac{\delta_{x}+\delta_{-x}}{2 x^{2}} .
\end{aligned}
$$


On unique solutions to martingale problems

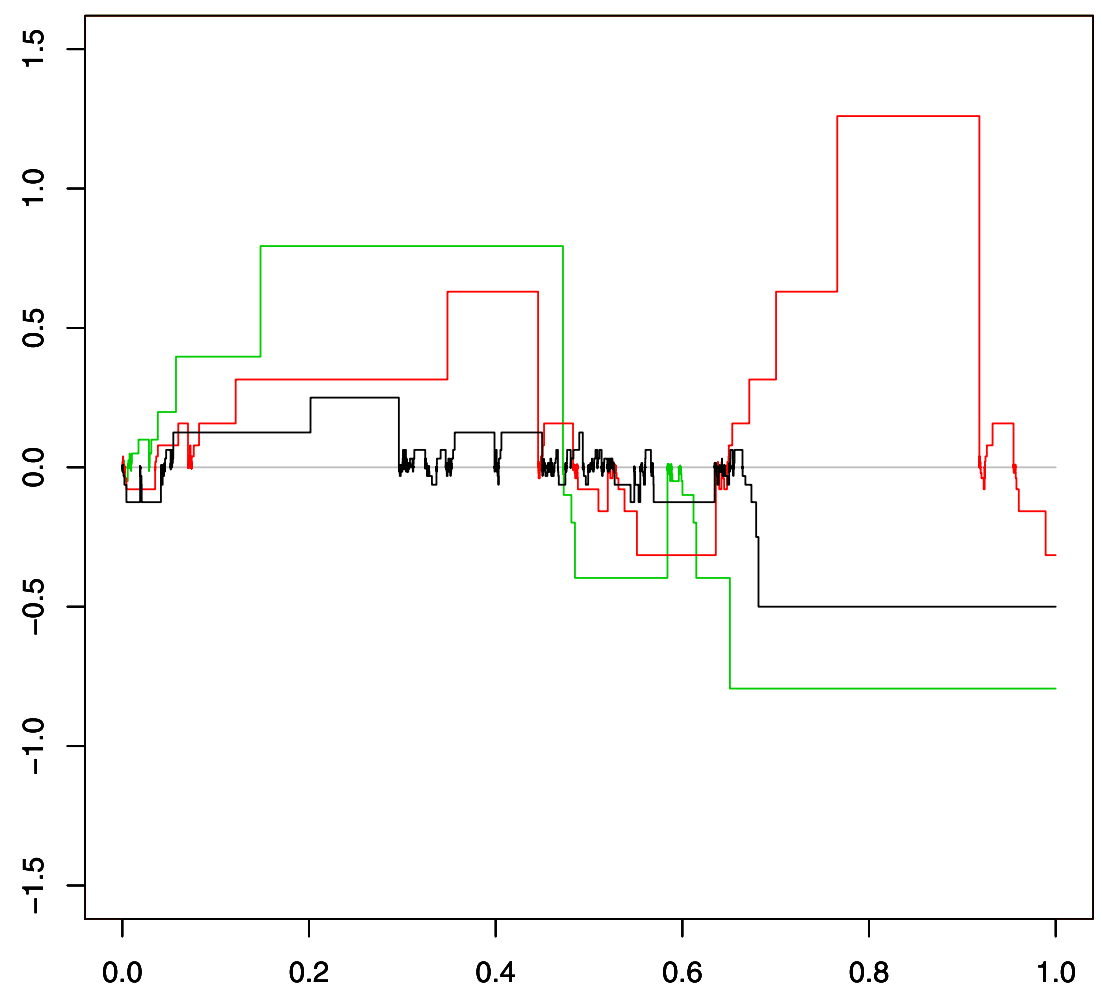

Figure 1: The picture shows simulated paths from three different Markov processes with symbol (3.1). All of them are based on the approximate generator $\mathcal{A}_{k, n}$ in the proof of Example 3.1 with $n=10$ and $k=1$ (black line), $k=\sqrt[3]{2}$ (red line) resp. $k=\sqrt[3]{4}$ (green line).

Then $(b, c, F)$ is the corresponding triplet in the sense of Remark 2.2. For any $n \in \mathbb{N}, k>0$ we also define

$$
q_{k, n}(x, u):= \begin{cases}q(x, u) & \text { if }|x| \geq k 2^{-n} \\ 4^{n \frac{\cos \left(u k 2^{-n}\right)-1}{k^{2}}} & \text { otherwise. }\end{cases}
$$

Then $q_{k, n}$ is a continuous symbol and the associated linear operator is given by

$$
\mathcal{A}_{k, n} f(x)= \begin{cases}\frac{f(2 x)-2 f(x)+f(0)}{2 x^{2}} & \text { if }|x| \geq k 2^{-n}, \\ 4^{n} \frac{f\left(x+k 2^{-n}\right)-2 f(x)+f\left(x-k 2^{-n}\right)}{2 k^{2}} & \text { otherwise. }\end{cases}
$$

The symbol $q_{k, n}$ satisfies the requirements of Theorem 2.7 whence there is a solution $X_{k, n}$ to the martingale problem $\left(\mathcal{A}_{k, n}, \delta_{0}\right)$ in the sense of [9, Section 4.3]. Since

$$
\begin{aligned}
X_{k, n}(t)= & X_{k, n}(0)+\int_{0}^{t} b\left(X_{k, n}(s)\right) d s+X_{k, n}^{c}(t) \\
& +(x-\chi(x)) * \mu^{X_{k, n}}(t)+\chi(x) *\left(\mu^{X_{k, n}}-\nu^{X_{k, n}}\right)(t) \\
= & x * \mu^{X_{k, n}}(t)=\sum_{s \leq t} \Delta X_{k, n}(s)
\end{aligned}
$$

and since $\Delta X_{k, n}(t) \in\left\{ \pm X_{k, n}(t-), \pm k 2^{-n}\right\}$ by $(3.2,3.3)$, we conclude that $X_{k, n}$ takes values only in the closed set $M_{k}:=k\left\{ \pm 2^{z}: z \in \mathbb{Z}\right\} \cup\{0\}$. Moreover, for any real-valued Schwartz function $f$ we have uniform convergence $\mathcal{A}_{k, n} f \rightarrow \mathcal{A} f$ for $n \rightarrow \infty$, where $\mathcal{A}$ denotes the operator associated with $q$. Using the proof of Lemma 5.2 and Chebyshev's 
inequality, we have that the sequence $\left(X_{n}\right)$ satisfies inequality (5.2) in [9, Remark 4.5.2]. The set of Schwartz functions is an algebra which separates points. By [9, Lemma 4.5.1, Remark 4.5.2] there is a subsequence of $\left(X_{k, n}\right)_{n \in \mathbb{N}}$ which converges weakly to a solution $X_{k}$ of the martingale problem $\left(\mathcal{A}, \delta_{0}\right)$. Note that $X_{k}$ takes values only in $M_{k}$.

Both $X_{1}, X_{\sqrt{2}}$ are solutions to the martingale problem related to $\mathcal{A}$ and initial law $\delta_{0}$, where $X_{1}$ takes values only in $M_{1}$ and $X_{\sqrt{2}}$ only in $M_{\sqrt{2}}$. Their extended generator in the sense of [5, Definition 2.3] is defined for all polynomials. Polynomials are mapped to polynomials of at most the same degree, which means that $X_{1}, X_{\sqrt{2}}$ are polynomial processes by [5, Theorem 2.10]. [5, Theorem 2.7] and its proof yields the moments. In particular, $E\left(X_{1}(t)^{2}\right)=E\left(X_{\sqrt{2}}(t)^{2}\right)>0$ for any $t>0$, which means that $X_{1}(t), X_{\sqrt{2}}(t)$ are not concentrated in zero. Since $M_{1} \cap M_{\sqrt{2}}=\{0\}$, this implies that $X_{1}, X_{\sqrt{2}}$ cannot have the same law.

We now turn to a related example with analytic symbol where uniqueness fails. It corresponds to an increasing process. It is once more polynomial in the sense of [5, Definition 2.1] or [11, Definition 2.1]. However, since its state space equals $\mathbb{R}_{+}$, it is not perfectly in line with the setup of this paper.

Example 3.2. Let

$$
q: \mathbb{R}_{+} \times \mathbb{R} \rightarrow \mathbb{C}, \quad(x, u) \mapsto \begin{cases}\frac{e^{i u x}-1}{x} & \text { for } x \neq 0, \\ i u & \text { otherwise }\end{cases}
$$

This function clearly has an entire extension to $\mathbb{C} \times \mathbb{C}$. There are solutions $X, Y$ to the $q$-martingale problem which start in 0 and are singular in the same sense as in the previous example. More generally, there are strong Markov processes $X$ and $Y$ with values in $\mathbb{R}_{+}$and symbol $q$, which do not have the same law. Once more $X, Y$ are polynomial processes. Starting in 0 , their $n$-th moment at time $t$ is given by

$$
E_{0}\left(X^{n}(t)\right)=E_{0}\left(Y^{n}(t)\right)=\frac{t^{n}}{n !} \prod_{k=1}^{n}\left(2^{k}-1\right), \quad t \geq 0, n \in \mathbb{N} .
$$

Exponent $q(x, \cdot)$ has Lévy-Khintchine triplet $\left(\chi(x) / x, 0, \delta_{x} / x\right)$ for $x>0$ and $(1,0,0)$ for $x=0$. Finally, observe that the continuous continuation given by

$$
\tilde{q}(x, u):=1_{\{x \geq 0\}} q(x, u)+1_{\{x<0\}} i u, \quad x, u \in \mathbb{R}
$$

on state space $\mathbb{R}$ yields the same process.

Proof. For any $n \in \mathbb{N}, k \in \mathbb{R}_{+}$and $x, u \in \mathbb{R}$ we define the approximation

$$
q_{k, n}(x, u):= \begin{cases}q(x, u) & \text { for } x \in\left[k 2^{-n}, k 2^{n}\right], \\ q\left(k 2^{-n}, u\right) & \text { for } x<k 2^{-n}, \\ q\left(k 2^{n}, u\right) & \text { for } x>k 2^{n} .\end{cases}
$$

of the symbol $q$. The claim follows now similarly as in Example 3.1.

\section{The symbol and the uniqueness problem}

The obvious question to ask is what conditions are needed to ensure uniqueness of the $q$-martingale problem for a given symbol $q$. For continuous processes the situation is well understood, The fact that SDE's have unique solutions under Lipschitz conditions directly yields uniqueness for $C^{2}$-symbols without jump part. 


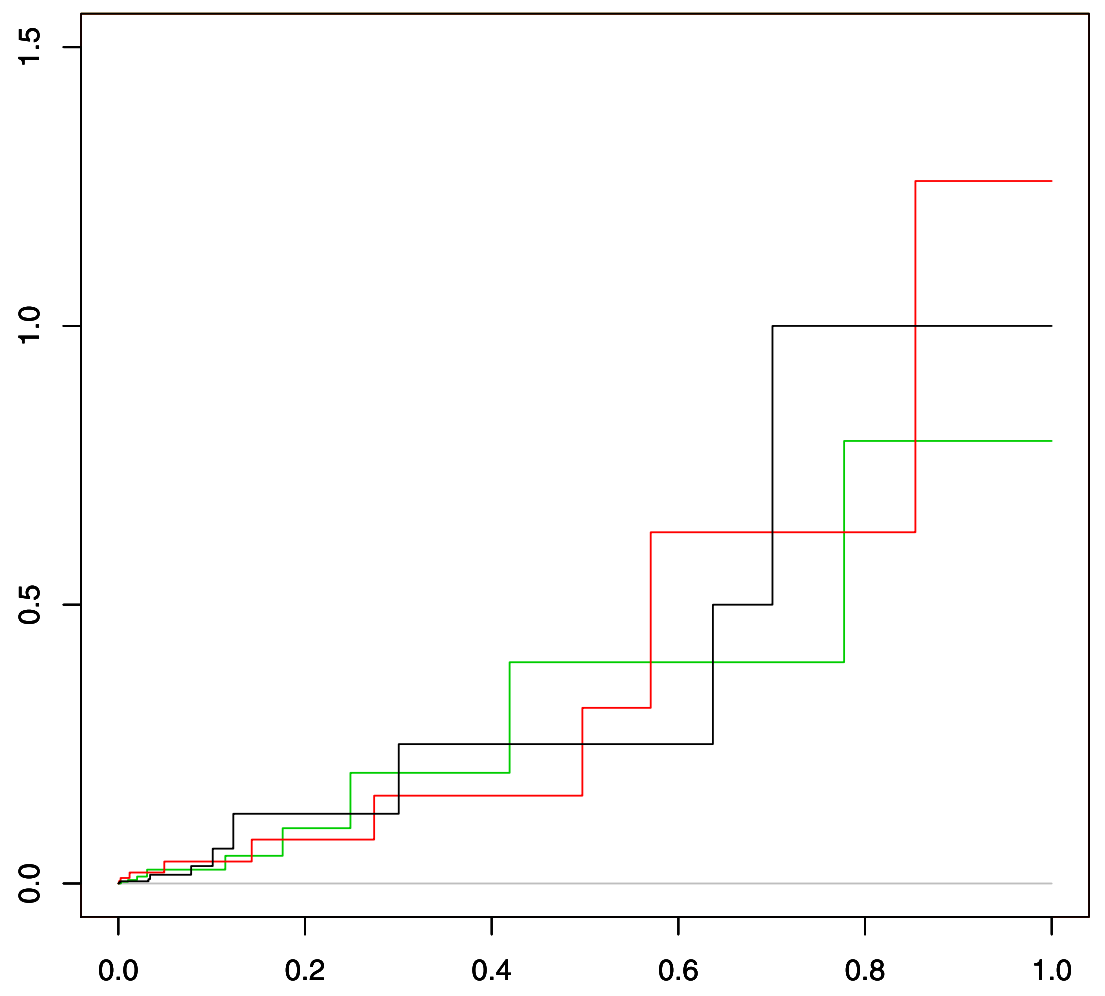

Figure 2: The picture shows simulated paths from three different Markov processes. Each of them uses the approximate generator $\mathcal{A}_{k, n}$ appearing in the proof of Example 3.2 with $k=1$ (black line), $k=\sqrt[3]{2}$ (red line) resp. $k=\sqrt[3]{4}$ (green line) and $n=10$ for all of them.

Proposition 4.1 (no jumps). Let $q \in C^{2}\left(\mathbb{R}^{d} \times \mathbb{R}^{d}, \mathbb{C}\right)$ be a symbol which has the representation $q(x, u)=i u b(x)-\frac{1}{2} u c(x) u$ for functions $b: \mathbb{R}^{d} \rightarrow \mathbb{R}^{d}, c: \mathbb{R}^{d} \rightarrow \mathbb{R}^{d \times d}$. Then uniqueness holds for the $q$-martingale problem.

Proof. Observe that $c$ takes actually values in the set of positive semidefinite matrices. Let $\sigma(x)$ be the positive square root of $c(x)$ for all $x \in \mathbb{R}^{d}$. Then [28, Theorem V.12.12] implies that $\sigma$ is Lipschitz. [28, Theorem V.12.1 and Section V.22] applied to $(b, \sigma)$ yields the claim.

As Example 3.1 indicates, the previous theorem does not hold for processes with jumps. One could express $q$-martingale problems for general symbols $q$ in terms of an SDE, but it is not clear what conditions on $q$ warrant Lipschitz continuity of the corresponding coefficients. Stroock-Varadhan type results, however, require the diffusion part of the symbol to be non-singular. A systematic study of existence and uniqueness property has been undertaken by Hoh in a number of papers. In [16, Theorem 5.7] he shows that a real-valued symbol satisfying similar conditions as in Theorem 4.4 below belongs to a unique strong Markov process. Hoh's result [15, Theorem 5.7] requires the likewise real-valued symbol to be infinitely smooth in both variables and an ellipticity condition to hold. His extension [17, Theorem 1.1] shows that uniqueness still holds if a state-depend power is applied to a symbol as in [15, Theorem 5.7]. Finally, see the related article [18, Theorem 9.4].

One of the main contributions of the present paper is the following uniqueness result. 
Theorem 4.2 (Uniqueness). Let $q \in C\left(\mathbb{R}^{d} \times \mathbb{R}^{d}, \mathbb{C}\right)$ be a Hölder-continuous symbol satisfying the requirements of Theorem 2.7. Moreover, suppose that there are $K \in \mathbb{R}_{+}$ and complex measures $\left(P_{t, u}\right)_{t \in[0,1], u \in \mathbb{R}^{d}}$ such that

1. $\widehat{P_{t, u}}(x):=\int e^{i v x} P_{t, u}(d v)=e^{t q(x, u)}$ for all $x \in \mathbb{R}^{d}$,

2. $\int \frac{1+|u+v|^{2}}{1+|u|^{2}}\left|P_{t, u}\right|(d v) \leq 1+K t$.

Then existence and uniqueness holds for the $q$-martingale problem. In particular, there is a unique Feller process starting in any given distribution and having symbol $q$.

Proof. The proof is to be found in Section 5.2.

Example 4.3. Let $q(x, u):=(1-\cos (x)) \psi(u), x, u \in \mathbb{R}$ where $\psi$ is a real-valued Lévy exponent on $\mathbb{R}$ which satisfies the requirements of Theorem 2.7, e.g. $\psi(u)=\frac{-u^{2}}{2}$. In particular, $q(0, u)=0$ for any $u \in \mathbb{R}$. Then existence and uniqueness for the $q$-martingale problem hold.

Proof. Instead of verifying the conditions in the previous theorem directly, we refer to Lemma 5.14 below, which follows from Theorem 4.2.

If the measure $P_{t, u}$ in Theorem 4.2 happens to be nonnegative as in Example 3.1, we have

$$
\int \frac{1+|u+v|^{2}}{1+|u|^{2}} P_{t, u}(d v) \leq 1+\frac{2|u|}{1+|u|^{2}}\left|\int v P_{t, u}(d v)\right|+\frac{1}{1+|u|^{2}} \int|v|^{2} P_{t, u}(d v) .
$$

In this case condition (2) in Theorem 4.2 can be interpreted as first and second moment condition on $P_{t, u}$, which can be vaguely viewed as a "smoothness" condition on $q$. However, in particular for complex $P_{t, u}$ it is less obvious how restrictive condition (2) is and how one can verify it. We therefore provide a second uniqueness result which follows from Theorem 4.2, but which is stated directly in terms of $q$.

Theorem 4.4. Let $q$ be a continuous symbol with $q(\cdot, u) \in C^{[d / 2]+3}\left(\mathbb{R}^{d}, \mathbb{C}\right)$ for all $u \in \mathbb{R}^{d}$ and such that $q$ satisfies the requirements of Theorem 2.7, cf. also Corollary 2.9. Let $\varphi: \mathbb{R}^{d} \rightarrow \mathbb{C}$ be a characteristic exponent satisfying the following conditions

$$
\begin{aligned}
|\operatorname{Re}(q(x, u))| & \geq g_{1}(x)|\varphi(u)|, \\
\left|\partial_{x}^{\alpha} q(x, u)\right| & \leq g_{2}(x)|\varphi(u)|
\end{aligned}
$$

for some bounded functions $g_{1}, g_{2}: \mathbb{R}^{d} \rightarrow(0, \infty)$ and any $\alpha \in \mathbb{N}^{d}$ with $|\alpha| \leq[d / 2]+3$. Then existence and uniqueness holds for the $q$-martingale problem.

Proof. The proof is to be found in Section 5.2.

Condition (4.2) is a uniform smoothness requirement. Condition (4.1), however, means that the symbol is bounded from below in an appropriate sense. Such an ellipticity condition occurs in the Stroock-Varadhan existence and uniqueness result, cf. [30, Theorem 4.3]. The advantage of the result in [30] is that continuity suffices and no extra smoothness is needed. Moreover, the drift only needs to be measurable. However, Stroock requires an ellipticity condition with respect to the explicit symbol $\varphi(u)=-\frac{1}{2} u^{2}$, which means that a continuous diffusion part is present everywhere. His proof also uses some extra regularity for the jump measure which, however, could be relaxed.

Since Stroock and Varadhan have published their result, some variants of the StroockVaradhan theorem with a more general ellipticity condition have been established by several authors, i.e. with a more general function $\varphi$ than for the original result, cf. 
On unique solutions to martingale problems

[20] for an overview. A recent result is due to Böttcher, cf. [2, Theorem 2.8], who requires equations $(4.1,4.2)$ for arbitrary $\alpha$ and, moreover, a certain boundedness for the derivatives with respect to $u$. Theorem 4.4 may be easier to apply in practice because it involves only finitely many derivatives and no smoothness in $u$.

\section{Proofs}

\subsection{Proof of the existence theorem}

The proof of Theorem 2.7 relies on the general statements [9, Theorem 4.5.4] and [9, Theorem 4.5.19], which yield existence of a solution and its strong Markov property, respectively. In order for the solution to be conservative we need to verify that explosion cannot happen. This is done with the help of Lemma 5.2, which yields finite second moments. These finite second moments are also needed for the verification of the conditions in [9, Theorems 4.5.11(b), 4.5.19], which are stated as Lemma 5.3 in our context.

We start with the semimartingale property of solutions, cf. [31, Corollary 2.6] for a more general setup.

Proposition 5.1 (Semimartingale characteristics). Let $q$ be a symbol and $X$ a solution to the q-martingale problem. Then $X$ is a semimartingale which allows for local or differential characteristics in the sense of [22, Definition 2.2]. Moreover, $X$ is quasi-left continuous, i.e. $\Delta X_{T}=0$ almost surely for any finite predictable stopping time $T$. If $q$ is represented by triplet $(b, c, F)$ as in Definition 2.1, then

$$
(\omega, t) \mapsto(b(X(t-)), c(X(t-)), F(X(t-), \cdot))(\omega)
$$

is a version of the local characteristics of $X$ relative to truncation function $\chi$.

Proof. Except for quasi-left continuity this follows from the definition and [21, Theorem II.2.42]. Quasi-left continuity is obtained from [21, Proposition II.2.9(i)].

We continue with a lemma which yields a sufficient condition for the existence of second moments.

Lemma 5.2. Let $q$ be a symbol which satisfies the requirements of Theorem 2.7. Moreover, let $X$ be a solution to the $q$-martingale problem. Then $E\left(\sup _{s \in[0, t]}|X(s)-X(0)|^{2}\right)<$ $\infty$ for any $t \in \mathbb{R}_{+}$.

Proof. Proposition 5.1 implies that $X$ is a semimartingale with local characteristics of the form (5.1). Boundedness of $g$ implies that it is a special semimartingale, cf. e.g. [21, Proposition II.2.29(a)]. The finite variation part $A$ in its canonical decomposition $X=X(0)+M+A$ is of the form

$$
A(t)=\int_{0}^{t}\left(b(X(s-))+\int(x-\chi(x)) F(X(s-), d x)\right) d s
$$

and hence bounded on any compact interval. For the local martingale part $M$ we have

$$
\left\langle M_{i}, M_{i}\right\rangle(t)=\int_{0}^{t}\left(c_{i i}(X(s-))+\int x_{i}^{2} F(X(s-), d x)\right) d s, \quad i=1, \ldots, d,
$$

which is bounded on any compact interval as well. Doob's inequality yields that

$$
E\left(\sup _{s \in[0, t]}\left|M_{i}(s)\right|^{2}\right) \leq 4 E\left(\left[M_{i}, M_{i}\right](t)\right)=4 E\left(\left\langle M_{i}, M_{i}\right\rangle(t)\right)<\infty, \quad i=1, \ldots, d
$$

for any $t \in \mathbb{R}_{+}$, which yields the claim. 
On unique solutions to martingale problems

Lemma 5.3. We assume that the requirements of [9, Theorems 4.5.11(b) and 4.5.19] hold in our setup. Specifically, let $\mathcal{A}_{0} \subset \bar{C}\left(\mathbb{R}^{d}\right) \times \bar{C}\left(\mathbb{R}^{d}\right)$ and denote by $\Gamma$ the set of solutions to the martingale problem for $\mathcal{A}_{0}$, i.e. all laws on the Skorokhod space $\mathbb{D}$ such that $f(X(t))-\int_{0}^{t} g(X(s)) d s$ is a martingale for any $(f, g) \in \mathcal{A}_{0}$, where $X$ denotes the canonical process. Writing $\mathcal{D}\left(\mathcal{A}_{0}\right):=\left\{f:(f, g) \in \mathcal{A}_{0}\right.$ for some $\left.g \in \mathcal{A}_{0}\right\}$, suppose that the closure $\overline{\mathcal{D}\left(\mathcal{A}_{0}\right)}$ contains an algebra that separates points and vanishes nowhere. Moreover, we assume $\Gamma_{\nu} \neq \varnothing$ for any probability measure $\nu$ on $\mathbb{R}^{d}$, where $\Gamma_{\nu}:=\left\{P \in \Gamma: P^{X(0)}=\nu\right\}$. Finally, suppose that for any compact set $K \subset \mathbb{R}^{d}$ and any $\epsilon, T \in(0, \infty)$ there exists a compact set $K^{\prime} \subset \mathbb{R}^{d}$ such that

$$
P\left(X(t) \in K^{\prime} \text { for all } t<T, X(0) \in K\right) \geq(1-\epsilon) P(X(0) \in K)
$$

for any $P \in \Gamma$.

Then there are measures $\left(P_{x}\right)_{x \in \mathbb{R}^{d}}$ in $\Gamma$ with $X(0)=x$ a.s. under $P_{x}, x \in \mathbb{R}^{d}$ and such that $x \mapsto P_{x}(X(t) \in A)$ is measurable for any $t \geq 0$ and any Borel set $A \subset \mathbb{R}^{d}$. Moreover, $\left(X,\left(P_{x}\right)_{x \in \mathbb{R}^{d}}\right)$ is strong Markov. Finally, if the $q$-martingale problem has several solutions for some initial law $P^{X(0)}=\nu$, then there are several such families of measures $\left(P_{x}\right)_{x \in \mathbb{R}^{d}}$.

Proof. Step 1: Let $\mathcal{A}$ be an extension of $\mathcal{A}_{0}$ as in [9, Theorem 4.5.19(a)]. Moreover, let $P_{x} \in \Gamma_{\delta_{x}}$ for $x \in \mathbb{R}^{d}$ such that $P_{x}$ solves the $\mathcal{A}$-martingale problem, which exists by [9, Theorem 4.5.19(c)]. We define the law $P(x, t, d y):=P_{x}(X(t) \in d y)$ for $x \in \mathbb{R}^{d}$, $t \geq 0$. Then $P(x, 0, \cdot)=\delta_{x}$. Let $f: \mathbb{R}^{d} \rightarrow \mathbb{R}$ be continuous and bounded, which implies that $t \mapsto f(X(t))$ is right-continuous and bounded. By dominated convergence we have that $t \mapsto E_{x} f(X(t))$ is right-continuous as well. Since $x \mapsto E_{x}(f(X(t)))$ is measurable, $(x, t) \mapsto E_{x} f(X(t))$ is measurable by [21, I.1.21 and I.1.26], applied to the right-continuous process $Y(x, t):=E_{x} f(X(t))$. Since this holds for any continuous bounded $f$, we conclude that $(x, t) \mapsto P(x, t, A)=P_{x}(X(t) \in A)$ is measurable for any $A \in \mathcal{B}\left(\mathbb{R}^{d}\right)$.

Let $s, t \geq 0$. [9, Theorem 4.5.19(d)] yields

$$
\begin{aligned}
P(t+s, x, A) & =P_{x}(X(s+t) \in A) \\
& =E_{x}\left(E_{x}\left(1_{A}(X(s+t)) \mid \mathscr{F}_{t}\right)\right) \\
& =E_{x}\left(P_{X(t)}(X(s) \in A)\right) \\
& =\int P_{y}(X(s) \in A) P_{x}^{X(t)}(d y) \\
& =\int P(y, s, A) P(x, t, d y)
\end{aligned}
$$

and hence $P$ is a transition function in the sense of [9, Page 156].

Step 2: From [9, Theorem 4.5.19(d)] we also get that $X$ is a strong Markov process in the sense of [9, Page 158] with transition function $P$. Indeed, let $P$ be a solution to the $\mathcal{A}$-martingale problem, $\tau$ a finite stopping time and $C \in \mathcal{B}(\mathbb{D})$. Then we have $E\left(1_{\{X((\tau \wedge n)+\cdot) \in C\}} \mid \mathscr{F}_{\tau \wedge n}\right)=P_{X(\tau \wedge n)}(C)$ for any $n \in \mathbb{N}$ by [9, Theorem 4.5.19(d)] because $\tau \wedge n$ is bounded. For the strong Markov property we have to show that equality holds for $\tau$ instead of $\tau \wedge n$. Clearly, $P_{X(\tau \wedge n)}(C) \rightarrow P_{X(\tau)}(C)$ pointwise for $n \rightarrow \infty$. Moreover, $Y_{n}:=1_{\{X((\tau \wedge n)+\cdot) \in C\}} \rightarrow Y_{\infty}:=1_{\{X(\tau+\cdot) \in C\}}$ pointwise and hence in $L^{2}(P)$ for $n \rightarrow \infty$. We obtain

$$
E\left(Y_{n}-Y_{\infty} \mid \mathscr{F}_{\tau \wedge n}\right)+E\left(Y_{\infty} \mid \mathscr{F}_{\tau \wedge n}\right)=E\left(Y_{n} \mid \mathscr{F}_{\tau \wedge n}\right) \rightarrow P_{X(\tau)}(C)
$$

and $E\left(Y_{n}-Y_{\infty} \mid \mathscr{F}_{\tau \wedge n}\right) \rightarrow 0$ in $L^{2}(P)$ as $n \rightarrow \infty$ because

$$
E\left(\left|E\left(Y_{n}-Y_{\infty} \mid \mathscr{F}_{\tau \wedge n}\right)\right|^{2}\right) \leq E\left(\left|Y_{n}-Y_{\infty}\right|^{2}\right) \rightarrow 0 .
$$


Moreover, $E\left(Y_{\infty} \mid \mathscr{F}_{\tau \wedge n}\right)=E\left(E\left(Y_{\infty} \mid \mathscr{F}_{\tau}\right) \mid \mathscr{F}_{\tau \wedge n}\right) \rightarrow E\left(Y_{\infty} \mid \mathscr{F}_{\tau}\right)$ in $L^{2}(P)$ when $n \rightarrow \infty$. Consequently,

$$
P_{X(\tau)}(C)=E\left(Y_{\infty} \mid \mathscr{F}_{\tau}\right) .
$$

Step 3: Now we turn to the last part of the claim. Here, we assume that $\Gamma_{\nu}$ contains more than one element for some Borel measure $\nu$ on $\mathbb{R}^{d}$. The proof of [9, Lemma 4.5.19] actually constructs extensions $\mathcal{A} \neq \mathcal{B}$ given in [9, Lemma 4.5.19(b)] which are maximal in the sense that any further extension of $\mathcal{A}$ or $\mathcal{B}$ does not meet [9, Lemma 4.5.19(c)] any more.

Then, $\mathcal{B} \nsubseteq \mathcal{A}$ due to maximality. Thus there is $(f, g) \in \mathcal{B}$ with $(f, g) \notin \mathcal{A}$. Assume by contradiction that $M(t):=f(X(t))-\int_{0}^{t} g(X(s)) d s$ is a $P_{x}$-martingale for any $x \in \mathbb{R}^{d}$. Then

$$
\mathcal{A}_{+}:=\{(h+\lambda f, k+\lambda g):(h, k) \in \mathcal{A}, \lambda \in \mathbb{R}\}
$$

is a strict extension of $\mathcal{A}$ which is a linear operator and such that the canonical process $X$ solves the $\mathcal{A}_{+}$-martingale problem under $P_{x}$ for any $x \in \mathbb{R}^{d}$. [9, Proposition 4.3.5] yields that $\mathcal{A}_{+}$is dissipative. This contradicts the maximality of $\mathcal{A}$. We conclude that there is $x \in \mathbb{R}^{d}$ such that $M$ is not a $P_{x}$-martingale. However, $M$ is a $Q_{x}$-martingale if the canonical process $X$ is a solution to the $\mathcal{B}$-martingale problem under $Q_{x}$ for any $x \in \mathbb{R}^{d}$. Consequently, $P_{x} \neq Q_{x}$.

Proof of Theorem 2.7. Step 1: Let $f$ be a real-valued Schwartz function and $\epsilon>0$. Boundedness of $g$ implies

$$
\sup _{x \in \mathbb{R}^{d}} F\left(x,\left\{y \in \mathbb{R}^{d}:|y| \geq a\right\}\right)<\epsilon
$$

for some sufficiently large $a>0$ such that the support of $\chi$ is contained in $B(0, a)$. Lemma 2.5 yields

$$
\begin{aligned}
|\mathcal{A} f(x)| & \leq|\nabla f(x)|\left|b(x)+\int\left(y 1_{B(0, a)}(y)-\chi(y)\right) F(x, d y)\right| \\
& +\frac{1}{2} \sup _{y \in B(x, a)}|H f(y)|\left(\operatorname{Tr}(c(x))+\int_{B(0, a)}|y|^{2} F(x, d y)\right) \\
& +\int_{B(0, a)^{c}}|f(x+y)-f(x)| F(x, d y) \\
& \leq\left(|\nabla f(x)|+\sup _{y \in B(x, a)}|H f(y)|\right) g(x)+2 \sup _{y \in \mathbb{R}^{d}}|f(y)| \epsilon \\
& \rightarrow 2 \sup _{y \in \mathbb{R}^{d}}|f(y)| \epsilon
\end{aligned}
$$

for any $x \in \mathbb{R}^{d}$. Hence $\mathcal{A} f(x) \rightarrow 0$ for $x \rightarrow \infty$.

Define $\tilde{c}(x):=c(x)+\int \chi(y) \chi(y)^{\top} F(x, d y)$. [21, Theorem VII.2.9] yields continuity of $b, \tilde{c}$ and of $x \mapsto \int h(y) F(x, d y)$ for any bounded continuous function $h: \mathbb{R}^{d} \rightarrow \mathbb{R}$ which vanishes in a neighbourhood of zero. Lemma 2.5 and linearity of the trace imply

$$
\begin{aligned}
\mathcal{A} f(x)= & \nabla f(x) b(x)+\frac{1}{2} \operatorname{Tr}(H f(x) \tilde{c}(x)) \\
& +\int\left(f(x+y)-f(x)-\nabla f(x) \chi(y)-\frac{1}{2} \operatorname{Tr}\left(H f(x) \chi(y) \chi(y)^{T}\right)\right) F(x, d y)
\end{aligned}
$$

for any $x \in \mathbb{R}^{d}$. Thus $x \mapsto \mathcal{A} f(x)$ is continuous. Together, this yields statement (3a). Moreover, the indirect implication of statement (2) follows from Lemma 2.6. 
Step 2: Let $f$ be any real-valued Schwartz function with maximum attained at $x_{0} \in \mathbb{R}^{d}$, i.e. $\sup _{x \in \mathbb{R}^{d}} f(x)=f\left(x_{0}\right)$. Then $\nabla f\left(x_{0}\right)=0$ and $H f\left(x_{0}\right)$ is negative semidefinite. Therefore

$$
\mathcal{A} f\left(x_{0}\right)=\frac{1}{2} \operatorname{Tr}\left(H f\left(x_{0}\right) c\left(x_{0}\right)\right)+\int\left(f\left(x_{0}+h\right)-f\left(x_{0}\right)\right) F\left(x_{0}, d h\right) \leq 0,
$$

i.e., $\mathcal{A}$ satisfies the positive maximum principle in the sense of $[9, \mathrm{p} .165]$, whence statement (3b) holds. Moreover, it is defined on a dense subset of $\hat{C}\left(\mathbb{R}^{d}\right)$ because its domain are the real-valued Schwartz functions.

Step 3: Let $\varphi: \mathbb{R}^{d} \rightarrow[0,1]$ be an infinitely differentiable function which is constantly 1 on the unit ball in $\mathbb{R}^{d}$ and whose support is contained in the centered ball with radius 2 . For any $n \in \mathbb{N}$ define the real-valued Schwartz function

$$
f_{n}: \mathbb{R}^{d} \rightarrow \mathbb{R}, \quad x \mapsto \varphi(x / n) .
$$

Then $f_{n} \rightarrow 1$ pointwise and the second derivatives of $f_{n}$ are bounded by $k / n^{2}$, where $k$ is a common bound for the first two partial derivatives of $\varphi$. Step 2 yields

$$
\mathcal{A} f_{n}(x)=\int\left(f_{n}(x+y)-1\right) F(x, d y)
$$

for $x \in \mathbb{R}^{d}$ and $n>|x|$. Due to a remainder estimate for the Taylor series we have

$$
\left|f_{n}(x+y)-1\right| \leq \frac{k|y|^{2}}{2 n^{2}}
$$

Thus the dominated convergence theorem yields

$$
\left|\mathcal{A} f_{n}(x)\right| \leq \int \frac{k|y|^{2}}{2 n^{2}} F(x, d y) \rightarrow 0, \quad n \rightarrow \infty,
$$

whence $\mathcal{A} f_{n}(x) \rightarrow 0$ pointwise. Similar arguments yield $\left|\mathcal{A} f_{n}(x)\right| \leq K g(x)$ for any $x \in \mathbb{R}^{d}$, $n \in \mathbb{N}$ and some constant $K>0$ which does not depend on $x$ and $n$. Thus we have $f_{n} \rightarrow 1$ and $\mathcal{A} f_{n} \rightarrow 0$ for $n \rightarrow \infty$, where the convergence holds relative to the bp-topology, cf. [9, p. 111]. This implies statement (3c). [9, Theorem 4.5.4 and Remark 4.5.5] yield that for any probability measure $\mu$ on $\mathbb{R}^{d}$ there is a solution to the martingale problem $(\mathcal{A}, \mu)$ in the sense of [9, Section 4.3].

Step 4: In order to show that there are solutions to the $q$-martingale problem, let $\mu$ be a probability measure and $X$ a solution to the martingale problem $(\mathcal{A}, \mu)$ in the sense of [9, Section 4.3]. Moreover, let $u \in \mathbb{R}^{d}$ and $\varphi: \mathbb{R}^{d} \rightarrow[0,1]$ as in Step 3. Define

$$
f_{n}(x):=e^{i u x} \varphi(x / n), \quad x \in \mathbb{R}^{d}, n \in \mathbb{N} .
$$

Similarly as in Step 3 one shows that there is a bound $B<\infty$ such that $\left|f_{n}(x)\right| \leq 1$, $\left|\mathcal{A} f_{n}(x)\right| \leq B$ for any $x \in \mathbb{R}^{d}, n \in \mathbb{N}$, and

$$
\begin{aligned}
f_{n}(x) & \rightarrow e^{i u x}, \\
\mathcal{A} f_{n}(x) & \rightarrow q(x, u) e^{i u x}
\end{aligned}
$$

for any $x \in \mathbb{R}^{d}$. Thus

$$
M_{f_{n}}(t) \stackrel{n \rightarrow \infty}{\longrightarrow} M_{u}(t):=e^{i u X(t)}-\int_{0}^{t} e^{i u X(s)} q(X(s), u) d s
$$

a.s. for any $t \in \mathbb{R}_{+}$. By dominated convergence, $M_{u}$ is a martingale which shows that $X$ is a solution to the $q$-martingale problem. Altogether, we obtain both the direct implication of statement (2) and statement (1). 
Step 5: The set of real-valued Schwartz functions is an algebra that separates points. Lemma 5.2 yields that that [9, (4.5.33)] holds for all choices of $K, \epsilon$ and $T$ where $K^{\prime}$ can be chosen according to Chebyshev's inequality. Thus [9, Theorem 4.5.11(b)] implies that the requirements of [9, Theorem 4.5.19] are met for $\Gamma:=\left\{P_{\mu}: P_{\mu}\right.$ solves $(\mathcal{A}, \mu)$ for some probability measure $\mu$ on $\left.\mathbb{R}^{d}\right\}$. Lemma 5.3 now yields statements (4) and (5).

Step 6: Let $x, u \in \mathbb{R}^{d}$ and $\left(P_{x}\right)_{x \in \mathbb{R}^{d}}$ measures on the canonical space such that the canonical process $X$ is a solution to the $q$-martingale problem with $X(0)=x P_{x}$-a.s. for any $x \in \mathbb{R}^{d}$. Then

$$
E_{x}\left(e^{i u X(t)}\right)=e^{i u x}+E_{x}\left(\int_{0}^{t} e^{i u X(s)} q(X(s), u) d s\right), \quad t \geq 0 .
$$

Hence right-continuity of $X$ in 0 yields

$$
\begin{aligned}
\frac{E_{x}\left(e^{i u(X(t)-x)}\right)-1}{t} & =\frac{1}{t} \int_{0}^{t} E_{x}\left(e^{i u(X(s)-x)} q(X(s), u) d s\right. \\
& \rightarrow E_{x}\left(e^{i u(X(0)-x)} q(X(0), u)\right. \\
& =q(x, u)
\end{aligned}
$$

for $t \downarrow 0$, which is statement (6).

\subsection{Proof of the uniqueness theorems}

The remainder of the paper is devoted to the proof of Theorems 4.2 and 4.4. The idea is as follows. We aim at proving uniqueness of univariate marginals in the Fourier domain, i.e. we show that for two solutions $X, Y$ the characteristic functions $\varphi_{X(t)}, \varphi_{Y(t)}$ coincide. This will be done by a Grönwall argument. We proceed in two steps. First we show that any solution can be approximated locally by a conditional Lévy process, cf. Lemmas 5.7, 5.8. Secondly we try to find bounds for the deviation rate of two piecewise Lévy processes, which leads to Theorem 4.2. In order to derive Theorem 4.4 , the conditions are first verified for simple symbols. Moreover, the set of symbols meeting the requirements has a certain closedness property, cf. Lemma 5.11. Then we can deduce Lemma 5.14 which states that uniqueness holds if the symbol can be locally approximated with a Fourier series satisfying some positivity condition. Finally, we construct such a Fourier series for elliptic symbols, cf. Lemma 5.15.

The localisation procedure for martingale problems reveals that uniqueness is a local property, cf. [9, Section 4.6] and [3, Theorem 3.28]. We restate a localisation theorem suitable for our applications with slightly different assumptions than the very related [3, Theorem 3.28]; the proof however is basically the same.

Proposition 5.4. Let $q$ be a symbol such that existence holds for the $q$-martingale problem. Let $\mathcal{U}$ be an open covering for $\mathbb{R}^{d}$ and for all $U \in \mathcal{U}$ let $q_{U}$ be a symbol such that

1. $q(x, u)=q_{U}(x, u)$ for any $x \in U, u \in \mathbb{R}^{d}$,

2. existence and uniqueness holds for the $q_{U}$-martingale problem,

3. $q(\cdot, u)$ is bounded for any $u \in \mathbb{R}^{d}$ and

4. $q_{U}(\cdot, u)$ is bounded for any $u \in \mathbb{R}^{d}, U \in \mathcal{U}$.

Then existence and uniqueness hold for the $q$-martingale problem.

Proof. W.l.o.g. we may assume that $\mathcal{U}$ is countable. Let $\mu$ be a probability measure on $\mathbb{R}^{d}$ and $U \in \mathcal{U}$. Define

$$
\begin{aligned}
\mathcal{B} & :=\left\{\left(e^{i u \cdot}, q(\cdot, u) e^{i u \cdot}\right): u \in \mathbb{R}^{d}\right\} \\
\mathcal{B}_{U} & :=\left\{\left(e^{i u \cdot}, q_{U}(\cdot, u) e^{i u \cdot}\right): u \in \mathbb{R}^{d}\right\}
\end{aligned}
$$


for any $U \in \mathcal{U}$. Observe that a stochastic càdlàg process is a solution to the $\mathcal{B}$-martingale problem if and only if it is a solution to the $q$-martingale problem. In particular, existence holds for the $\mathcal{B}$-martingale problem. Let $U \in \mathcal{U}$ and observe that a stochastic process is a solution to the stopped martingale problem $(\mathcal{B}, \mu, U)$ if and only if it is a solution to the stopped martingale problem $\left(\mathcal{B}_{U}, \mu, U\right)$ in the sense of [9, Page 216].

Moreover, [9, Theorem 4.6.1] yields that the stopped martingale problem $\left(\mathcal{B}_{U}, \mu, U\right)$ has unique solutions. Hence $(\mathcal{B}, \mu, U)$ has unique solutions for any $U \in \mathcal{U}$. [9, Theorem 4.6.2] yields uniqueness for the martingale problem $(\mathcal{B}, \mu)$. Since $\mu$ was arbitrary, we have uniqueness for the $q$-martingale problem.

The next result is a Grönwall-type theorem with perturbation which will be useful later.

Lemma 5.5. Let $I=[0, T], c \in(0, \infty)$, and $\beta: \mathbb{R}_{+} \rightarrow \mathbb{R}_{+}$such that $\lim _{t \rightarrow 0} \beta(t) / t=0$. Let $\varphi: \mathbb{R}_{+} \rightarrow \mathbb{R}_{+}$such that for all $s, t \in I$ with $s<t$ we have

$$
\varphi(t) \leq(1+(t-s) c) \varphi(s)+\beta(t-s) .
$$

Then $\varphi(t) \leq \varphi(0) e^{c t}$ for all $t \in I$. In particular, $\varphi=0$ if $\varphi(0)=0$.

Proof. Let $t \in \mathbb{R}_{+}$and $N \in \mathbb{N}$. The inequality above yields

$$
\varphi\left(t \frac{n+1}{N}\right) \leq(1+t c / N) \varphi\left(t \frac{n}{N}\right)+\beta(t / N)
$$

for $n=0, \ldots, N-1$. Hence

$$
\varphi(t) \leq(1+t c / N)^{N} \varphi(0)+\sum_{k=0}^{N-1}(1+t c / N)^{k} \beta(t / N) .
$$

Since $(1+t c / N)^{N} \leq \exp (t c)$, we have

$$
\varphi(t) \leq \exp (t c) \varphi(0)+\sum_{k=0}^{N-1}(1+t c / N)^{k} \beta(t / N) .
$$

The geometric series sums up to

$$
\sum_{k=0}^{N-1}(1+t c / N)^{k}=\frac{(1+t c / N)^{N}-1}{t c / N} \leq N \frac{e^{t c}-1}{t c} .
$$

However, $N \beta(t / N)$ converges to 0 for $N \rightarrow \infty$. Hence

$$
\sum_{k=0}^{N-1}(1+t c / N)^{k} \beta(t / N) \rightarrow 0 .
$$

We conclude $\varphi(t) \leq \exp (t c) \varphi(0)$ as desired.

In the sequel we will work with the norm

$$
\|\cdot\|: \hat{C}\left(\mathbb{R}^{d}, \mathbb{C}\right) \rightarrow \mathbb{R}_{+}, \quad \varphi \mapsto \sup \left\{\frac{|\varphi(u)|}{1+|u|^{2}}: u \in \mathbb{R}^{d}\right\} .
$$

Remark 5.6. A sequence of characteristic functions which converges with respect to $\|\cdot\|$, converges uniformly on compact sets. Lévy's continuity theorem [19, Theorem 19.1] yields weak convergence of the corresponding sequence of random variables. 
As mentioned above, the proof of Theorem 4.2 is based on local comparison to conditional Lévy processes. If we consider a symbol $q$, a solution $X$ to the $q$-martingale problem, $s \geq 0$, and an $\mathscr{F}_{s}$-conditional Lévy process $L$ with Lévy exponent $\psi(u)=q(X(s), u)$, i.e. $\mathrm{E}\left(e^{i u\left(L\left(t_{2}\right)-L\left(t_{1}\right)\right)} \mid \mathscr{F}_{s}\right)=\exp \left(\left(t_{2}-t_{1}\right) \psi(u)\right)$, then the following lemma measures the difference $\mathrm{E}(\Gamma(t, s, u))$ between the characteristic functions of $X(t)$ and $L(t)-L(s)+X(s)$ in terms of an expected path integral over $q(X, u)$. However, the conditional Lévy process $L$ appears in the statement and proof only through its characteristic funtion.

Lemma 5.7 (Comparison to conditional Lévy process I). Let $q$ be a continuous symbol such that $q(\cdot, u)$ is bounded for all $u \in \mathbb{R}^{d}$. Moreover, let $X$ be a solution to the $q$ martingale problem and for $s \geq 0$ let $Q_{s}$ be a regular version of the conditional law of $X$ given $\mathscr{F}_{s}$, i.e.

- $Q_{s}: \Omega \times \mathcal{B}\left(D_{\mathbb{R}^{d}}[0, \infty)\right) \rightarrow[0,1]$ is a transition kernel from $\left(\Omega, \mathscr{F}_{s}, P\right)$ to $\mathrm{D}$ and

- $E\left(f(X) \mid \mathscr{F}_{s}\right)=\int f(\rho) Q_{s}(d \rho)$ for any bounded measurable function $f: \mathbb{D} \rightarrow \mathbb{C}$.

Define

$$
\Gamma(t, s, u):=\int_{s}^{t} e^{(t-r) q(X(s), u)} \int_{\mathbb{D}} e^{i u \rho(r)}(q(\rho(r), u)-q(\rho(s), u)) Q_{s}(d \rho) d r
$$

for $s, t \in \mathbb{R}_{+}, u \in \mathbb{R}^{d}$. Then we have

$$
\varphi_{X}(t, u)=E(\exp ((t-s) q(X(s), u)+i u X(s)))+E(\Gamma(t, s, u))
$$

for any $t, s \in \mathbb{R}_{+}, u \in \mathbb{R}^{d}$ with $s<t$, where $\varphi_{X}(t, u):=E\left(e^{i u X(t)}\right)$. Moreover,

$$
|E \Gamma(t, s, u)| \leq \int_{s}^{t} E|q(X(r), u)-q(X(s), u)| d r
$$

for any $x, u \in \mathbb{R}^{d}, 0 \leq s \leq t<\infty$.

Proof. Let $s \in \mathbb{R}_{+}, u \in \mathbb{R}^{d}$. For all $t \in[s, \infty)$ we have

$$
\begin{aligned}
\varphi_{s}(t, u) & :=\int_{\mathbb{D}} e^{i u \rho(t)} Q_{s}(d \rho) \\
& =M_{u}(s)+\int_{\mathbb{D}} \int_{0}^{t} q(\rho(r), u) \exp (i u \rho(r)) d r Q_{s}(d \rho) \\
& =M_{u}(s)+\int_{0}^{s} q(X(r), u) \exp (i u X(r)) d r \\
& +\int_{s}^{t} \int_{\mathbb{D}} q(\rho(r), u) \exp (i u \rho(r)) Q_{s}(d \rho) d r \\
= & e^{i u X(s)}+\int_{s}^{t} \int_{\mathbb{D}} q(\rho(r), u) \exp (i u \rho(r)) Q_{s}(d \rho) d r .
\end{aligned}
$$

We can see that $t \mapsto \varphi_{s}(t, u)$ is $P$-a.s. continuous. Thus the canonical process on $\left(\mathbb{D}, \mathcal{B}(\mathbb{D}), Q_{s}(\omega, \cdot)\right)$ is weakly continuous for $P$-almost every $\omega \in \Omega$. Consequently,

$$
r \mapsto \int_{\mathbb{D}} q(\rho(r), u) \exp (i u \rho(r)) Q_{s}(\omega, d \rho)
$$

is continuous for $P$-almost every $\omega \in \Omega$. This shows that $t \mapsto \varphi_{s}(t, u)$ is continuously differentiable for $P$-almost every $\omega \in \Omega$.

The fundamental theorem of calculus yields

$$
\begin{aligned}
\partial_{t} \varphi_{s}(t, u) & =\int_{\mathbb{D}} q(\rho(t), u) \exp (i u \rho(t)) Q_{s}(d \rho) \\
& =\varphi_{s}(t, u) q(X(s), u)+g(s, t)
\end{aligned}
$$


for all $t>s P$-a.e. where

$$
g(s, t):=\int_{\mathbb{D}} \exp (i u \rho(t))(q(\rho(t), u)-q(\rho(s), u)) Q_{s}(d \rho) .
$$

Moreover, we have $\varphi_{s}(s, u)=e^{i u X(s)}$. The variation of constants formula [13, Satz 98.5] implies

$$
\begin{aligned}
\varphi_{s}(t, u) & =e^{(t-s) q(X(s), u)+i u X(s)}+\int_{s}^{t} e^{(t-r) q(X(s), u)} g(s, r) d r \\
& =e^{(t-s) q(X(s), u)+i u X(s)}+\Gamma(t, s, u) .
\end{aligned}
$$

Thus we obtain

$$
\begin{aligned}
E\left(e^{i u X(t)}\right) & =E\left(\varphi_{s}(t, u)\right) \\
& =E\left(e^{(t-s) q(X(s), u)+i u X(s)}\right)+E(\Gamma(t, s, u)) .
\end{aligned}
$$

Finally, we have

$$
\begin{aligned}
|E(\Gamma(t, s, u))| & \leq E\left(\int_{s}^{t} \int_{\mathbb{D}}|(q(\rho(r), u)-q(\rho(s), u))| Q_{s}(d \rho) d r\right) \\
& =\int_{s}^{t} E(|(q(X(r), u)-q(X(s), u))|) d r
\end{aligned}
$$

for any $t \geq s$.

Lemma 5.8 (Comparison to conditional Lévy process II). Let $q$ be a Hölder continuous symbol. Moreover, let $I \subset \mathbb{R}_{+}$be a bounded interval and $X$ a solution to the $q$-martingale problem. Then there is a function $\beta: \mathbb{R}_{+} \rightarrow \mathbb{R}_{+}$such that $\lim _{t \rightarrow 0} \beta(t) / t=0$ and

$$
\int_{s}^{t} \frac{E|q(X(r), u)-q(X(s), u)|}{1+|u|^{2}} d r \leq \beta(t-s)
$$

for all $s, t \in I, u \in \mathbb{R}^{d}$ with $s<t$.

Proof. Let $f$ be a bounded and continuous function such that

$$
|q(x, u)-q(y, u)| \leq f(x-y)\left(1+|u|^{2}\right), \quad x, y, u \in \mathbb{R}^{d} .
$$

Then

$$
\frac{E|q(X(r), u)-q(X(s), u)|}{1+|u|^{2}} \leq E(f(X(r)-X(s))) .
$$

Proposition 5.1 states that $X$ is quasi-left continuous. Hence

$$
H: \mathbb{R}_{+} \times \mathbb{R}_{+} \rightarrow \mathbb{R}_{+},(r, s) \rightarrow E(f(X(r)-X(s)))
$$

is continuous and $H(s, s)=0$ for all $s \in \mathbb{R}_{+}$. The mean value theorem theorem yields the claim for $\beta(t):=t \sup \{H(r, s): r, s \in I$ and $|r-s| \leq t\}$.

We can now show that the univariate marginals of solutions to the martingale problem are uniquely determined under certain conditions.

Lemma 5.9. Let $q \in C\left(\mathbb{R}^{d} \times \mathbb{R}^{d}, \mathbb{C}\right)$ be a continuous and Hölder-continuous symbol. Moreover, let $K \in \mathbb{R}_{+}$and $I=\left[0, t_{0}\right]$ for some $t_{0}>0$. Assume that for any $t \in I, u \in \mathbb{R}^{d}$ there is a complex measure $P_{t, u}$ such that 
1. $\widehat{P_{t, u}}(x)=e^{t q(x, u)}$ for all $x \in \mathbb{R}^{d}$ and

2. $\int \frac{1+|u+v|^{2}}{1+|u|^{2}}\left|P_{t, u}\right|(d v) \leq 1+K t$.

If $X, Y$ are solutions to the $q$-martingale problem with the same initial distribution, then $X(t)$ and $Y(t)$ have the same distribution for all $t \in I$.

Proof. Observe that condition (2) implies that the total variation measure $\left|P_{t, u}\right|$ is finite. Define $d(t):=\left\|\varphi_{X}(t, \cdot)-\varphi_{Y}(t, \cdot)\right\|$ for all $t \in \mathbb{R}_{+}$where $\varphi_{X}(t, \cdot)$ and $\varphi_{Y}(t, \cdot)$ denote the characteristic functions of $X(t)$ resp. $Y(t)$. Let $g_{t, u}(x):=e^{t q(x, u)+i u x}$. Lemmas 5.7, 5.8 yield

$$
d(t) \leq \sup \left\{\frac{\left|E\left(g_{t-s, u}(X(s))\right)-E\left(g_{t-s, u}(Y(s))\right)\right|}{1+|u|^{2}}: u \in \mathbb{R}^{d}\right\}+\beta(t-s)
$$

for all $s, t \in I$ with $s<t$, where $\beta$ is a function as in Lemma 5.8 with $\lim _{t \rightarrow 0} \beta(t) / t=0$. Moreover, condition (1) and Fubini's theorem imply

$$
\begin{aligned}
E\left(g_{t-s, u}(X(s))\right) & =\iint e^{i v X(s)} P_{t-s, u}(d v) e^{i u X(s)} d P \\
& =\int \varphi_{X}(s, u+v) P_{t-s, u}(d v)
\end{aligned}
$$

and likewise for $Y$. We obtain

$$
\begin{aligned}
d(t) & \leq d(s) \sup \left\{\int \frac{1+|u+v|^{2}}{1+|u|^{2}}\left|P_{t-s, u}\right|(d v): u \in \mathbb{R}^{d}\right\}+\beta(t-s) \\
& \leq d(s)(1+K(t-s))+\beta(t-s) .
\end{aligned}
$$

By Lemma 5.5 we have $d(t)=0$ for all $t \in I$. Thus the characteristic functions of $X(t)$ and $Y(t)$ coincide, whence they have the same law.

Corollary 5.10. Assume that the requirements of Lemma 5.9 are fulfilled and that existence holds for the $q$-martingale problem. Then uniqueness holds for the $q$-martingale problem.

Proof. Let $X, Y$ be solutions with the same initial law and let $T \in[0, \infty]$ be maximal such that $X(t), Y(t)$ have the same law for all $t \in[0, T)$. By Proposition $5.1 X$ and $Y$ are quasi-left continuous, which implies that $X(T)$ and $Y(T)$ have the same law. Assume by contradiction that $T \neq \infty$. Then $\widetilde{X}(t):=X(T+t), \widetilde{Y}(t):=Y(T+t)$ are solutions to the $q$-martingale problem with the same initial law. Lemma 5.9 yields that $\widetilde{X}, \widetilde{Y}$ have the same one-dimensional distribution up to $t_{0}$ and hence $X, Y$ have the same univariate marginals up to $T+t_{0}$. This contradicts the maximality of $T$. Hence [9, Theorem 4.4.2] yields the claim.

Proof of Theorem 4.2. Theorem 2.7 implies existence and Corollary 5.10 yields uniqueness. The second statement follows from Proposition 2.10.

We now turn to the proof of Theorem 4.4 .

Lemma 5.11. Let $q$ be a symbol. Consider $q_{n}: \mathbb{R}^{d} \times \mathbb{R}^{d} \rightarrow \mathbb{C}$, complex measures $P_{t, u, n}$ on $\mathbb{R}^{d}$, and $K_{n} \geq 0$ such that

1. $\widehat{P}_{t, u, n}(x)=\exp \left(t q_{n}(x, u)\right)$,

2. $\left|P_{t, u, n}\right|\left(\mathbb{R}^{d}\right) \leq 1$,

3. $\int v\left|P_{t, u, n}\right|(d v)=0$,

4. $\int|v|^{2}\left|P_{t, u, n}\right|(d v) \leq t K_{n}\left(1+|u|^{2}\right)$,

5. $q(x, u)=\sum_{n \in \mathbb{N}} q_{n}(x, u)$, and 
6. $K:=\sum_{n=1}^{\infty} K_{n}<\infty$

for any $n \in \mathbb{N}, x, u \in \mathbb{R}^{d}, t \in[0,1]$. Then there are complex measures $P_{t, u}$ on $\mathbb{R}^{d}$ such that

1. $\widehat{P}_{t, u}(x)=\exp (t q(x, u))$,

2. $\int \frac{1+|u+v|^{2}}{1+|u|^{2}}\left|P_{t, u}\right|(d v) \leq 1+K t$

for any $x, u \in \mathbb{R}^{d}, t \in[0,1]$.

Proof. Let $t \in[0,1], u \in \mathbb{R}^{d}$. Observe that $\left(\otimes_{n=1}^{l}\left|P_{t, u, n}\right|\left(\left(\mathbb{R}^{d}\right)^{l}\right)\right)_{l \in \mathbb{N}}$ is a decreasing sequence and denote its limit by $c \in[0,1]$. Note that

$$
\begin{aligned}
c & =\lim _{l \rightarrow \infty} \prod_{n=1}^{l}\left|P_{t, u, n}\right|\left(\mathbb{R}^{d}\right) \\
& \geq\left|\lim _{l \rightarrow \infty} \prod_{n=1}^{l} P_{t, u, n}\left(\mathbb{R}^{d}\right)\right| \\
& =\left|\lim _{l \rightarrow \infty} \exp \left(t \sum_{n=1}^{l} q_{n}(x, u)\right)\right| \\
& =\exp (t \operatorname{Re}(q(x, u))) \\
& >0 .
\end{aligned}
$$

For $a_{n}:=P_{t, u, n}\left(\mathbb{R}^{d}\right)=\exp \left(t q_{n}(0, u)\right)$, assumption (5) yields $\Pi_{n=1}^{\infty} a_{n}=\exp (t q(0, u)) \in \mathbb{C}$.

By (4), (6) and Proposition B.6 the infinite convolutions $P_{t, u}$ of $\left(P_{t, u, n}\right)_{n \in \mathbb{N}}$ and $Q_{t, u}$ of $\left(\left|P_{t, u, n}\right|\right)_{n \in \mathbb{N}}$ exist and we have $\left|P_{t, u}\right| \leq Q_{t, u}$ in the sense that the density is bounded by one. Moreover,

$$
\begin{aligned}
\int e^{i v x} P_{t, u}(d v) & =\lim _{l \rightarrow \infty} \int e^{i v x}\left(P_{t, u, 1} * \cdots * P_{t, u, l}\right)(d v) \\
& =\lim _{l \rightarrow \infty} \exp \left(t \sum_{n=1}^{l} q_{n}(x, u)\right) \\
& =\exp (t q(x, u))
\end{aligned}
$$

for any $x \in \mathbb{R}^{d}$. Hence, $P_{t, u}$ satisfies (1).

By Proposition B.4 the infinite product measure $\bar{P}_{t, u}$ of $\left(P_{t, u, n}\right)_{n \in \mathbb{N}}$ exists. Let $\pi_{n}$ : $\left(\mathbb{R}^{d}\right)^{\mathbb{N}} \rightarrow \mathbb{R}^{d},\left(x_{l}\right)_{l \in \mathbb{N}} \mapsto x_{n}$. Then we have

$$
\begin{aligned}
\left.\left|\int\right| \pi_{n}\right|^{2} d \bar{P}_{t, u} \mid & \leq \int\left|\pi_{n}\right|^{2} d\left|\bar{P}_{t, u}\right| \\
& =\frac{c}{\left|P_{t, u, n}\right|\left(\mathbb{R}^{d}\right)} \int v^{2}\left|P_{t, u, n}\right|(d v) \\
& \leq t K_{n}\left(1+|u|^{2}\right)
\end{aligned}
$$

and

$$
\begin{aligned}
\int \pi_{n} \pi_{m} d\left|\bar{P}_{t, u}\right| & =\frac{c}{\left|P_{t, u, n}\right|\left(\mathbb{R}^{d}\right)\left|P_{t, u, m}\right|\left(\mathbb{R}^{d}\right)} \int v\left|P_{t, u, n}\right|(d v) \int w\left|P_{t, u, m}\right|(d w) \\
& =0
\end{aligned}
$$


where we used (3) and (4). We conclude that

$$
\begin{aligned}
\int\left|\sum_{n=1}^{l} \pi_{n}\right|^{2} d\left|\bar{P}_{t, u}\right| & =\int \sum_{n=1}^{l}\left|\pi_{n}\right|^{2} d\left|\bar{P}_{t, u}\right| \\
& \leq \sum_{n=1}^{l} t K_{n}\left(1+|u|^{2}\right) \\
& \leq t K\left(1+|u|^{2}\right) .
\end{aligned}
$$

This implies

$$
\int v^{2} Q_{t, u}(d v)=\lim _{l \rightarrow \infty} \int\left(\sum_{n=1}^{l} \pi_{n}\right)^{2} d\left|\bar{P}_{t, u}\right| \leq t K\left(1+|u|^{2}\right) .
$$

Similar arguments show that $Q_{t, u}\left(\mathbb{R}^{d}\right) \leq 1$ and $\int v Q_{t, u}(d v)=0$. Thus we have

$$
\begin{aligned}
\int \frac{1+|u+v|^{2}}{1+|u|^{2}}\left|P_{t, u}\right|(d v) & \leq \int \frac{1+|u+v|^{2}}{1+|u|^{2}} Q_{t, u}(d v) \\
& \leq 1+t K
\end{aligned}
$$

as desired.

Recall from Theorem 4.2 that uniqueness holds for symbol $q$ in Lemma 5.11 if it satisfies the requirements of Theorem 2.7. The functions $q_{n}$ will later be chosen from the following lemma.

Lemma 5.12. Let $n \in \mathbb{R}^{d}, a, b \in \mathbb{C}, k \in \mathbb{R}$ such that $\operatorname{Re}(a) \geq|b|$. Then there is a complex measure $P_{t}$ for any $t \in[0,1]$ such that

1. $\widehat{P}_{t}(x)=\exp (t(b \cos (k n x)-a))$,

2. $\left|P_{t}\right|\left(\mathbb{R}^{d}\right) \leq 1$,

3. $\int v\left|P_{t}\right|(d v)=0$,

4. $\int|v|^{2}\left|P_{t}\right|(d v) \leq t|b||k n|^{2}$.

Moreover, there is a complex measure $Q_{t}$ such that

1. $\widehat{Q_{t}}(x)=\exp (t(b \sin (k n x)-a))$,

2. $\left|Q_{t}\right|\left(\mathbb{R}^{d}\right) \leq 1$,

3. $\int v\left|Q_{t}\right|(d v)=0$,

4. $\int|v|^{2}\left|Q_{t}\right|(d v) \leq t|b||k n|^{2}$.

Proof. Let $\mu$ be a complex measure on $\mathbb{R}^{d}$ with total variation less or equal 1. Moreover, let $P_{t}:=\exp (-t a) \exp (t b \mu)$, cf. Appendix A. Then we have for all $x \in \mathbb{R}^{d}$

$$
\begin{aligned}
\widehat{P}_{t}(x) & =\exp (-t a) \exp (t b \hat{\mu}(x)) \\
\left|P_{t}\right| & \leq \exp (-t \operatorname{Re}(a)) \exp (t|b||\mu|), \\
\left|P_{t}\right|\left(\mathbb{R}^{d}\right) & \leq \exp (t(|b|-\operatorname{Re}(a))) \leq 1 \\
\int|v|^{2}\left|P_{t}\right|(d v) & \leq t|b|\left(\int|v|^{2}|\mu|(d v)+\left|\int v\right| \mu|(d v)|^{2}\right)
\end{aligned}
$$

for any $x \in \mathbb{R}^{d}$, where (5.2) means that the measure on the left is absolutely continuous with density at most one relative to the measure on the right. The last inequality follows from Lemma A.4. For the specific choice $\mu=\frac{1}{2}\left(\delta_{n k}+\delta_{-n k}\right)$, Lemma A.2 yields

$$
\int v\left|P_{t}\right|(d v)=0
$$


which shows the first assertion. For the specific choice $\mu=\frac{1}{2 i}\left(\delta_{n k}-\delta_{-n k}\right)$, Lemma A.3 implies

$$
\int v\left|P_{t}\right|(d v)=0
$$

and hence the second claim.

Lemma 5.13. Let $q \in C^{(1,0)}\left(\mathbb{R}^{d} \times \mathbb{R}^{d}, \mathbb{C}\right)$ be a symbol such that $(x, u) \mapsto \frac{q(x, u)}{1+|u|^{2}}$ and $(x, u) \mapsto \frac{\nabla_{1} q(x, u)}{1+|u|^{2}}$ are bounded. Then $q$ is ( $f$-)Hölder continuous for

$$
f(x):=\min \left\{2 \sup _{y, u \in \mathbb{R}^{d}} \frac{|q(y, u)|}{1+|u|^{2}},|x| \sup _{y, u \in \mathbb{R}^{d}} \frac{\left|\nabla_{1} q(y, u)\right|}{1+|u|^{2}}\right\}, \quad x \in \mathbb{R}^{d} .
$$

In particular, if $q$ satisfies the requirements of Theorem 4.4 for some $\varphi$, then $q$ is Höldercontinuous.

Proof. We have

$$
|q(x, u)-q(y, u)| \leq 2 \sup _{z, v \in \mathbb{R}^{d}} \frac{|q(z, v)|}{1+|v|^{2}}, \quad x, y, u \in \mathbb{R}^{d} .
$$

Moreover, the fundamental theorem of calculus yields

$$
|q(x, u)-q(y, u)| \leq|x-y| \sup _{z, v \in \mathbb{R}^{d}} \frac{\left|\nabla_{1} q(z, v)\right|}{1+|v|^{2}}, \quad x, y, u \in \mathbb{R}^{d}
$$

and hence

$$
|q(x, u)-q(y, u)| \leq f(x-y), \quad x, y, u \in \mathbb{R}^{d}
$$

as claimed.

Now assume that $q$ satisfies the requirements of Theorem 4.4. Equation (4.2) yields that $|q(x, u)| \leq g_{2}(x) \varphi(u)$ for some continuous function $g_{2}: \mathbb{R}^{d} \rightarrow \mathbb{R}_{+}$which is bounded by some constant $C_{1}<\infty$. Since $|\varphi(u)| \leq C_{2}\left(1+|u|^{2}\right)$ for some constant $C_{2}<\infty$, we get $|q(x, u)| \leq C_{1} C_{2}\left(1+|u|^{2}\right)$. Moreover, we have $\left|\partial_{x_{j}} q(x, u)\right| \leq g_{2}(x) \varphi(u)$ and hence $(x, u) \mapsto \frac{\nabla_{1} q(x, u)}{1+|u|^{2}}$ is bounded by $C$ where $C:=C_{1} C_{2} d$.

Lemma 5.14 (Fourier conditions). Let $q \in C^{(1,0)}\left(\mathbb{R}^{d} \times \mathbb{R}^{d}, \mathbb{C}\right)$ be a symbol with the following properties.

1. $q$ satisfies the requirements of Theorem 2.7 .

2. There is a constant $c>0$ such that $|q(x, u)|+\left|\nabla_{1} q(x, u)\right| \leq c\left(1+|u|^{2}\right)$ for all $x, u \in \mathbb{R}^{d}$.

3. It has Fourier series representation, i.e. there are $a_{n}(u), b_{n}(u) \in \mathbb{C}$ for all $n \in \mathbb{Z}^{d}$ and a constant $k>0$ such that

$$
q(x, u)=\sum_{n \in \mathbb{Z}^{d}}\left(a_{n}(u) \cos (k n x)+b_{n}(u) \sin (k n x)\right)
$$

and the family $\left(a_{n}, b_{n}\right)_{n \in \mathbb{Z}^{d}}$ satisfies:

(a) the real part of $-a_{0}(u)$ dominates the absolute sum of the other coefficients, i.e.

$$
-\operatorname{Re}\left(a_{0}(u)\right) \geq \sum_{n \in \mathbb{Z}^{d} \backslash\{0\}}\left(\left|a_{n}(u)\right|+\left|b_{n}(u)\right|\right)
$$

for all $u \in \mathbb{R}^{d}$ and 
(b)

$$
K:=|k|^{2} \sup \left\{\sum_{n \in \mathbb{Z}^{d}}|n|^{2}\left(\frac{\left|a_{n}(u)\right|}{1+|u|^{2}}+\frac{\left|b_{n}(u)\right|}{1+|u|^{2}}\right): u \in \mathbb{R}^{d}\right\}<\infty .
$$

Then existence and uniqueness holds for the q-martingale problem.

Proof. Lemma 5.13 states that $q$ is Hölder continuous.

Since the coefficient $b_{0}$ does not play any role in the representation of $q$ we may assume $b_{0}=0$. Let $u \in \mathbb{R}^{d}, t \in[0,1]$. Then the Fourier series can be rewritten as

$$
q(x, u)=\tilde{a}_{0}(u)+\sum_{n \in \mathbb{Z}^{d} \backslash\{0\}}\left(\left(a_{n}(u) \cos (k n x)-\left|a_{n}(u)\right|\right)+\left(b_{n}(u) \sin (k n x)-\left|b_{n}(u)\right|\right)\right),
$$

where $\tilde{a}_{0}(u):=a_{0}(u)+\sum_{n \in \mathbb{Z}^{d} \backslash\{0\}}\left(\left|a_{n}(u)\right|+\left|b_{n}(u)\right|\right)$ and $\operatorname{Re}\left(\tilde{a}_{0}(u)\right) \leq 0$. By Lemma 5.12 there are complex measures $P_{t, u, n}, Q_{t, u, n}$, such that

1. $\widehat{P_{t, u, n}}(x)=\exp \left(t\left(a_{n}(u) \cos (k n x)-\left|a_{n}(u)\right|\right)\right)$,

2. $\left|P_{t, u, n}\right|\left(\mathbb{R}^{d}\right) \leq 1$,

3. $\int v\left|P_{t, u, n}\right|(d v)=0$,

4. $\int|v|^{2}\left|P_{t, u, n}\right|(d v) \leq t\left|a_{n}(u)\right||k n|^{2}$

and

1. $\widehat{Q_{t, u, n}}(x)=\exp \left(t\left(b_{n}(u) \sin (k n x)-\left|b_{n}(u)\right|\right)\right)$,

2. $\left|Q_{t, u, n}\right|\left(\mathbb{R}^{d}\right) \leq 1$,

3. $\int v\left|Q_{t, u, n}\right|(d v)=0$,

4. $\int|v|^{2}\left|Q_{t, u, n}\right|(d v) \leq t\left|b_{n}(u)\right||k n|^{2}$

for all $n \in \mathbb{Z}^{d} \backslash\{0\}$. Moreover, the measure $P_{t, u, 0}:=\exp \left(t \tilde{a}_{0}(u)\right) \delta_{0}$ satisfies

1. $\widehat{P_{t, u, 0}}(x)=\exp \left(t \tilde{a}_{0}(u)\right)$,

2. $\left|P_{t, u, 0}\right|\left(\mathbb{R}^{d}\right)=\exp \left(t \operatorname{Re}\left(\tilde{a}_{0}(u)\right)\right) \leq 1$,

3. $\int v\left|P_{t, u, 0}\right|(d v)=0$,

4. $\int|v|^{2}\left|P_{t, u, 0}\right|(d v)=0$.

Lemmas 5.11 and 5.9 yield uniqueness of the solution to the $q$-martingale problem.

The Fourier conditions in Lemma 5.14 might seem hard to verify. However, ellipticity and Fourier ellipticity are almost equivalent as can be seen from the proof of the next lemma.

Lemma 5.15. Let $q$ be a continuous symbol such that $q(\cdot, u) \in C^{[d / 2]+3}\left(\mathbb{R}^{d}, \mathbb{C}\right)$ for all $u \in \mathbb{R}^{d}$ and such that

1. $q$ satisfies the requirements of the existence theorem 2.7 and

2. for every $x_{0}$ there is a neighbourhood $V$ of $x_{0}$ and $L<\infty$ such that $\psi:=q\left(x_{0}, \cdot\right)$ satisfies

$$
\begin{aligned}
\left|\partial_{x}^{\beta} q(x, u)\right| & \leq L|\operatorname{Re}(\psi(u))| \\
\left|\partial_{x}^{\alpha} q(x, u)\right| & \leq L\left(1+|u|^{2}\right)
\end{aligned}
$$

for all $x \in V, u \in \mathbb{R}^{d}, \beta, \alpha \in \mathbb{N}^{d}$ with $|\beta| \leq[d / 2]+1$ and $|\alpha| \leq[d / 2]+3$.

Then existence and uniqueness hold for the $q$-martingale problem. 
On unique solutions to martingale problems

Proof. Step 1: Set $m:=[d / 2]+1$ and let $u \in \mathbb{R}^{d}$. W.l.o.g. we may assume that $V$ is open, convex, and bounded. The first inequality together with the mean value theorem implies

$$
\left|q(x, u)-q\left(x_{0}, u\right)\right| \leq L\left|x-x_{0}\right||\operatorname{Re}(\psi(u))|
$$

for all $x \in V$. Let $\varphi \in C^{\infty}\left([0,1]^{d},[0,1]\right)$ such that it is constant 1 on $[1 / 4,3 / 4]^{d}$ and compactly supported in $(0,1)^{d}$. Set

$$
C_{\varphi}:=\sup \left\{\left|\partial^{\alpha} \varphi(x)\right|: \alpha \in \mathbb{N}^{d},|\alpha| \leq m+2, x \in \mathbb{R}^{d}\right\},
$$

let $\ell \geq 1$ be large enough such that the cube centered at $x_{0}$ of radius $1 / \ell$ is contained in $V$, and define

$$
q_{\ell}:[0,1]^{d} \times \mathbb{R}^{d} \rightarrow \mathbb{C},(y, u) \mapsto \varphi(y)\left(q\left(\gamma_{\ell}(y), u\right)-\psi(u)\right)+\psi(u),
$$

where $\gamma_{\ell}:[0,1]^{d} \rightarrow V, y \mapsto \frac{y-h}{\ell}+x_{0}$ and $h:=\left(\frac{1}{2}, \ldots, \frac{1}{2}\right)$.

Step 2: For $s \in\{1, \ldots, m\}$ we have

$$
\begin{aligned}
& \sup \left\{\left|\partial_{y}^{\beta} q_{\ell}(y, u)\right|: y \in[0,1]^{d}, \beta \in \mathbb{N}^{d},|\beta|=s\right\} \\
\leq & \sup \left\{\left|q\left(\gamma_{\ell}(y), u\right)-\psi(u)\right|\left|\partial_{y}^{\beta} \varphi(y)\right|: y \in[0,1]^{d}, \beta \in \mathbb{N}^{d},|\beta|=s\right\} \\
& +2^{m} C_{\varphi} \sup \left\{\left|\partial_{y}^{\beta}\left(q\left(\gamma_{\ell}(y), u\right)-\psi(u)\right)\right|: y \in[0,1]^{d}, \beta \in \mathbb{N}^{d}, 1 \leq|\beta| \leq s\right\} \\
\leq \quad & L C_{\varphi} \sup \left\{\left|\gamma_{\ell}(y)-x_{0}\right|: y \in[0,1]^{d}\right\}|\operatorname{Re}(\psi(u))| \\
& +2^{m} C_{\varphi} \frac{1}{\ell} \sup \left\{\left|\partial_{x}^{\beta}(q(x, u)-\psi(u))\right|: x \in V, \beta \in \mathbb{N}^{d}, 1 \leq|\beta| \leq s\right\} \\
\leq & K_{1} \frac{|\operatorname{Re}(\psi(u))|}{\ell}
\end{aligned}
$$

with $K_{1}:=\left(1+2^{m}\right) L C_{\varphi}$. By [12, Theorem 3.2.16] there is another constant $K_{2}<\infty$ such that

$$
\left\|q_{\ell}(\cdot, u)\right\|_{A(T)} \leq K_{2} \frac{|\operatorname{Re}(\psi(u))|}{\ell}
$$

where $\left\|q_{\ell}(\cdot, u)\right\|_{A(T)}=\sum_{n \in \mathbb{Z}^{d} \backslash\{0\}}\left|c_{n}(u)\right|$ is the absolute sum of the Fourier coefficients

$$
c_{n}(u):=\int_{[0,1]^{d}} q_{\ell}(x, u) e^{-2 \pi i n x} d x, \quad n \in \mathbb{Z}^{d}
$$

except for the coefficient $a_{0}(u):=c_{0}(u)$, which appears to be missing in the statement of [12, Theorem 3.2.16]. Thus there is $\ell \geq 2 L$ such that

$$
\left\|q_{\ell}(\cdot, u)\right\|_{A(T)} \leq \frac{|\operatorname{Re}(\psi(u))|}{4} .
$$

We also have

$$
\begin{aligned}
\left|\operatorname{Re}\left(a_{0}(u)\right)-\operatorname{Re}(\psi(u))\right| & \leq\left|a_{0}(u)-\psi(u)\right| \\
& \leq \int_{[0,1]^{d}}\left|q_{\ell}(y, u)-\psi(u)\right| d y \\
& \leq L|\operatorname{Re}(\psi(u))| \int_{[0,1]^{d}}\left|\gamma_{\ell}(y)-x_{0}\right| d y \\
& \leq L \frac{|\operatorname{Re}(\psi(u))|}{\ell} \\
& \leq \frac{|\operatorname{Re}(\psi(u))|}{2}
\end{aligned}
$$


which implies $|\operatorname{Re}(\psi(u))| \leq 2\left|\operatorname{Re}\left(a_{0}(u)\right)\right|$. For $n \in \mathbb{Z}^{d} \backslash\{0\}$ set

$$
\begin{aligned}
a_{n} & :=e^{2 \pi i n\left(h-\ell x_{0}\right)} c_{n}, \\
b_{n} & :=i e^{2 \pi i n\left(h-\ell x_{0}\right)} c_{n} .
\end{aligned}
$$

Then

$$
\begin{aligned}
\sum_{n \in \mathbb{Z}^{d} \backslash\{0\}}\left(\left|a_{n}(u)\right|+\left|b_{n}(u)\right|\right) & \leq 2 \sum_{n \in \mathbb{Z}^{d} \backslash\{0\}}\left|c_{n}(u)\right| \\
& =2\left\|q_{\ell}(\cdot, u)\right\|_{A(T)} \\
& \leq \frac{|\operatorname{Re}(\psi(u))|}{a n d, h 2} \\
& \leq\left|\operatorname{Re}\left(a_{0}(u)\right)\right| \\
& =-\operatorname{Re}\left(a_{0}(u)\right)
\end{aligned}
$$

which implies (5.4).

Step 3: (5.5) can be deduced by applying [12, Proposition 3.1.2(10)] and the same arguments as in Step 2 to $\Delta_{1} q$ and using (5.7) instead of (5.6).

Step 4: Let $U$ be the cube centered at $x_{0}$ with radius $1 /(4 \ell)$. For $x \in U$ with $y:=\gamma_{\ell}^{-1}(x)$ we have $y \in[1 / 4,3 / 4]^{d}$ and

$$
q_{\ell}(y, u)=\varphi(y)\left(q\left(\gamma_{\ell}(y), u\right)-\psi(u)\right)+\psi(u)=q(x, u)
$$

Define $\widetilde{q}_{x_{0}, \ell}(x, u):=\widetilde{q}_{\ell}\left(\gamma_{\ell}^{-1}(x), u\right), x \in \mathbb{R}^{d}$, where $\widetilde{q}_{\ell}(\cdot, u)$ denotes the periodic continuation of $q_{\ell}(\cdot, u)$ to $\mathbb{R}^{d}$. The inversion formula [12, Proposition 3.1.14] yields

$$
\begin{aligned}
\widetilde{q}_{x_{0}, \ell}(x, u) & =\widetilde{q}_{\ell}(y, u) \\
& =\sum_{n \in \mathbb{Z}^{d}} c_{n}(u) e^{2 \pi i n y} \\
& =\sum_{n \in \mathbb{Z}^{d}} c_{n}(u) e^{2 \pi i n\left(h-\ell x_{0}\right)} e^{2 \pi i \ell n x} \\
& =\sum_{n \in \mathbb{Z}^{d}}\left(a_{n}(u) \cos (2 \pi \ell n x)+b_{n}(u) \sin (2 \pi \ell n x)\right) \\
& =\sum_{n \in \mathbb{Z}^{d}}\left(a_{n}(u) \cos (k n x)+b_{n}(u) \sin (k n x)\right)
\end{aligned}
$$

where $k:=2 \pi \ell$. Thus (5.3) holds for $\widetilde{q}_{x_{0}, \ell}$.

Moreover, $\widetilde{q}_{\ell}(\cdot, u)$ satisfies the requirements of Theorem 2.7 and, together with Steps 2, 3, those of Lemma 5.14. The localisation theorem 5.4 yields that existence and uniqueness holds for the $q$-martingale problem.

Proof of Theorem 4.4. $q$ satisfies the requirements in Lemma 5.15.

\section{A Convolutions and total variation}

In this appendix we recall various properties of the total variation and the convolution of complex measures on $\mathcal{B}\left(\mathbb{R}^{d}\right)$. A complex measure on $\mathbb{R}^{d}$ is a function $\mu: \mathcal{B}\left(\mathbb{R}^{d}\right) \rightarrow \mathbb{C}$ such that $\mu\left(\cup_{A \in \mathcal{Z}} A\right)=\sum_{A \in \mathcal{Z}} \mu(A)$ for any countable family $\mathcal{Z} \subset \mathcal{B}\left(\mathbb{R}^{d}\right)$ of pairwise disjoint sets, cf. [6, Section §3.4]. We denote the set of complex measures on $\mathbb{R}^{d}$ by $\mathcal{C}\left(\mathbb{R}^{d}\right)$. A decomposition of a measurable set $A$ is a finite system $\mathcal{Z}$ of pairwise disjoint 
measurable sets such that $\cup_{B \in \mathcal{Z}} B=A$. The total variation measure of $\mu$ is the measure defined by

$$
|\mu|(A):=\sup \left\{\sum_{B \in \mathcal{Z}}|Q(B)|: \mathcal{Z} \text { is a decomposition of } A\right\}, \quad A \in \mathcal{B}\left(\mathbb{R}^{d}\right) .
$$

The total variation of $\mu$ is defined by $\|\mu\|:=|\mu|\left(\mathbb{R}^{d}\right)$. The product measure of complex measures $\mu, \nu$ on $\mathbb{R}^{d}$ resp. $\mathbb{R}^{n}$ is the complex measure $\mu \otimes \nu$ on $\mathbb{R}^{d} \times \mathbb{R}^{n}$ given by

$$
(\mu \otimes \nu)(A \times B)=\mu(A) \nu(B), \quad A \in \mathcal{B}\left(\mathbb{R}^{d}\right), B \in \mathcal{B}\left(\mathbb{R}^{n}\right) .
$$

The convolution of complex measures $\mu, \nu$ on $\mathbb{R}^{d}$ is the complex measure $\mu * \nu$ on $\mathbb{R}^{d}$ defined by

$$
(\mu * \nu)(A)=\int \mu(A-x) \nu(d x), \quad A \in \mathcal{B}\left(\mathbb{R}^{d}\right) .
$$

Complex measures $\mu, \nu$ on $\mathbb{R}^{d}$ are called orthogonal if there is $A \in \mathcal{B}\left(\mathbb{R}^{d}\right)$ such that $\mu(B)=0$ for any Borel set $B \subset A$ and $\nu(C)=0$ for any Borel set $C \subset \mathbb{R}^{d} \backslash A$. The Dirac measure concentrated in $a \in \mathbb{R}^{d}$ is denoted by $\delta_{a}$. The Fourier transform of a complex measure $\mu$ on $\mathbb{R}^{d}$ is the function $\hat{\mu}: \mathbb{R}^{d} \rightarrow \mathbb{C}$ given by

$$
\hat{\mu}(u):=\int e^{i\langle u, x\rangle} \mu(d x)
$$

A complex measure $\mu$ on $\mathbb{R}^{d}$ is symmetric (resp. anti-symmetric) if $\mu(A)=\mu(-A)$ (resp. $\mu(A)=-\mu(-A)$ ) for any $A \in \mathcal{B}\left(\mathbb{R}^{d}\right)$.

Let us recall several properties of complex measures, which can be found or easily derived from results in [6].

Lemma A.1. Let $\mu, \nu$ be complex measures on $\mathbb{R}^{d}$ and $\eta$ a complex measure on $\mathbb{R}^{n}$. Then the following statements hold.

1. $|\mu|$ is an $\mathbb{R}_{+}$-valued (and hence finite) measure.

2. $|\mu(A)| \leq|\mu|(A)$ for any $A \in \mathcal{B}\left(\mathbb{R}^{d}\right)$.

3. $\mu$ is a regular measure in the sense of [6, Definition \$15.2.1].

4. $\|\cdot\|$ is a complete norm on $\mathcal{C}\left(\mathbb{R}^{d}\right)$.

5. (Hahn-Jordan decomposition) There are $\mathbb{R}_{+}$-valued measures $\mu_{1}, \mu_{2}, \mu_{3}, \mu_{4}$ such that

$$
\mu=\left(\mu_{1}-\mu_{2}\right)+i\left(\mu_{3}-\mu_{4}\right),
$$

where $\mu_{1}, \mu_{2}$ are orthogonal and $\mu_{3}, \mu_{4}$ are orthogonal.

6. A measurable function $f: \mathbb{R}^{d} \rightarrow \mathbb{C}$ is $\mu$-integrable if and only if it is $|\mu|$-integrable and in that case

$$
\left|\int f d \mu\right| \leq \int|f| d|\mu|
$$

7. Any bounded measurable function $f: \mathbb{R}^{d} \rightarrow \mathbb{C}$ is $\mu$-integrable.

8. For any $\mu * \nu$-integrable function $f$ we have

$$
\int f(v)(\mu * \nu)(d v)=\iint f(v+w) \mu(v) \nu(w) .
$$

9. $|\mu * \nu|$ is absolutely continuous with respect to $|\mu| *|\nu|$ with density bounded by 1 .

10. $\|\mu * \nu\| \leq\|\mu\|\|\nu\|$

11. $|\mu \otimes \eta|=|\mu| \otimes|\eta|$ 
12. If $\mu$ and $\nu$ are orthogonal, then $|\mu+\nu|=|\mu|+|\nu|$.

13. $\left(\mathcal{C}\left(\mathbb{R}^{d}\right),+, *,\|\cdot\|\right)$ is a complex commutative Banach algebra with unit $\delta_{0}$.

14. The Fourier transform ${ }^{\wedge}$ is a one-to-one homomorphism of algebras which is continuous with respect to the total variation norm and the uniform norm, respectively.

15. $\mu$ is symmetric resp. anti-symmetric if its Fourier transform is a symmetric resp. anti-symmetric function.

For an introduction to analytic functional calculus see e.g. [26, Definition 3.3.1]. For a complex measure $\mu$ and a function $f: \mathbb{C} \rightarrow \mathbb{C}$ which is holomorphic on a neighbourhood of the spectrum $\sigma(\mu):=\left\{z \in \mathbb{C}: z \delta_{0}-\mu\right.$ is $*$-invertible $\}$ of $\mu$ we write $f(\mu)$ for the complex measure obtained by the analytic functional calculus applied to $\mu$ and $f$.

Lemma A.2. Let $\mu$ be a complex measure on $\mathbb{R}^{d}$. If $\mu$ is symmetric or anti-symmetric, then $|\mu|$ is symmetric. If $f: \mathbb{C} \rightarrow \mathbb{C}$ is holomorphic on a neighbourhood of the spectrum of $\mu$ and $\mu$ is symmetric, then $f(\mu)$ is symmetric. If $f$ is an odd entire function and $\mu$ is anti-symmetric, then $f(\mu)$ is anti-symmetric. If $f$ is an even entire function and $\mu$ is anti-symmetric, then $f(\mu)$ is symmetric.

Proof. Let $\mu$ be symmetric or anti-symmetric and $A \in \mathcal{B}\left(\mathbb{R}^{d}\right)$. Let $\epsilon>0$ and $\mathcal{Z}$ be a decomposition of $A$ such that

$$
|\mu|(A) \leq \epsilon+\sum_{B \in \mathcal{Z}}|\mu(B)| .
$$

Then

$$
|\mu|(A)-\epsilon \leq \sum_{B \in \mathcal{Z}}|\mu(-B)| \leq|\mu|(-A) .
$$

Hence $|\mu|(A) \leq|\mu|(-A) \leq|\mu|(A)$, which implies symmetry.

Let $\mu$ be symmetric, $f$ an entire function and $z \in \mathbb{C}$. Then $z \delta_{0}-\mu$ is symmetric. If $z$ is not in the spectrum of $\mu$, then the measure $R(z, \mu)$ with the property $\left(z \delta_{0}-\mu\right) * R(z, \mu)=\delta_{0}$ is symmetric as well. Thus

$$
f(\mu)=\frac{1}{2 \pi i} \int_{\Gamma} f(z) R(z, \mu) d z
$$

is symmetric as well, where $\Gamma$ is a suitable integration path.

Now let $\mu$ be anti-symmetric and $z \in \mathbb{C}$ outside of the spectrum of $\mu$. Then

$$
(R(z, \mu)-R(-z, \mu))^{\wedge}(u)=\frac{1}{z+\hat{\mu}(u)}+\frac{1}{z-\hat{\mu}(u)}, \quad u \in \mathbb{R}^{d} .
$$

By Lemma A.1(15) $\alpha_{z}:=R(z, \mu)-R(-z, \mu)$ is symmetric. Similar arguments yield that $\beta_{z}:=R(z, \mu)+R(-z, \mu)$ is anti-symmetric and we obviously have $R(z, \mu)=1 / 2\left(\alpha_{z}+\beta_{z}\right)$. Let $\Gamma$ be a symmetric path around the spectrum of $\mu$. Then

$$
f(\mu)=\frac{1}{4 \pi i} \int_{\Gamma} f(z) \alpha_{z} d z+\frac{1}{4 \pi i} \int_{\Gamma} f(z) \beta_{z} d z .
$$

Observe that the first summand is symmetric and the second summand is anti-symmetric. If $f$ is even, then the first summand vanishes and hence $f(\mu)$ is symmetric. If $f$ is odd, then the second summand vanishes and hence $f(\mu)$ is anti-symmetric.

Lemma A.3. Let $\mu=z\left(\delta_{a}-\delta_{-a}\right)$ for some $a \in \mathbb{R}^{d}, z \in \mathbb{C}$. Then $|\exp (\mu)|$ is symmetric. 
Proof. For $w \in \mathbb{C}$ with $|w|>2|z|$ define

$$
\begin{aligned}
\alpha_{w} & :=R(w, \mu)-R(-w, \mu), \\
\beta_{w} & :=R(w, \mu)+R(-w, \mu),
\end{aligned}
$$

where $R(w, \mu)$ is the complex measure such that $R(w, \mu) *\left(w \delta_{0}-\mu\right)=\delta_{0}$. From their Fourier transforms we conclude that that $\alpha_{w}$ is supported on $O:=\{k a: k \in \mathbb{Z}, k$ is odd $\}$ and $\beta_{w}$ is supported on $E:=\{k a: k \in \mathbb{Z}, k$ is even $\}$. By (A.1) and the subsequent observation, $\sinh (\mu)$ is concentrated on $O$ while $\cosh (\mu)$ is concentrated on $E$. Proposition A.1 yields

$$
|\exp (\mu)|=|\sinh (\mu)|+|\cosh (\mu)| .
$$

Lemma A.2 yields that $|\exp (\mu)|$ is the sum of symmetric measures and hence symmetric.

Lemma A.4. Let $\mu$ be a complex measure on $\mathbb{R}^{d}$ such that $\int|v|^{2}|\mu|(d v)<\infty$. Then

$$
\begin{aligned}
\exp (\mu)\left(\mathbb{R}^{d}\right) & =\exp \left(\mu\left(\mathbb{R}^{d}\right)\right), \\
\int v \exp (\mu)(d v) & =\int v \mu(d v) \exp \left(\mu\left(\mathbb{R}^{d}\right)\right), \\
\int|v|^{2} \exp (\mu)(d v) & \left.=\iint|v|^{2} \mu(d v)+\left|\int v \mu(d v)\right|^{2}\right) \exp \left(\mu\left(\mathbb{R}^{d}\right)\right)
\end{aligned}
$$

where existence of the integral on the left hand side of the third equality is implied by assumption.

Proof. The first claimed equality follows from Lemma A.1(8).

In order to show (A.3), we start by verifying that $\int|v|^{2}|\exp (\mu)|(d v)<\infty$, which implies that the integral on the left-hand side of (A.3) exists. To this end let $a:=|\mu|\left(\mathbb{R}^{d}\right)$, $b:=\int v|\mu|(d v)$ and $c:=\int|v|^{2}|\mu|(d v)$ be the zeroth, first, and second moment of the total variation measure $|\mu|$. We have

$$
\begin{aligned}
\int|v|^{2}|\exp (\mu)|(d v) & \leq \int|v|^{2} \exp (|\mu|)(d v) \\
& =\sum_{n=0}^{\infty} \frac{1}{n !} \int|v|^{2}\left|\mu^{n}\right|(d v) \\
& =\sum_{n=0}^{\infty} \frac{1}{n !} \int \cdots \int\left(\sum_{i=1}^{n}\left|v_{i}\right|^{2}+\sum_{i, j=1}^{n}\left\langle v_{i}, v_{j}\right\rangle\right)|\mu|\left(d v_{1}\right) \ldots|\mu|\left(d v_{n}\right) \\
& =\sum_{n=0}^{\infty} \frac{1}{n !}\left(n a^{n-1} c+n(n-1) a^{n-2}|b|^{2}\right) \\
& =\left(c+|b|^{2}\right) \exp (a) \\
& <\infty
\end{aligned}
$$

Repeating the equalities and defintions of $a, b, c$ with $\mu$ instead of $|\mu|$ yields (A.3).

Equation (A.2) follows easily similarly.

\section{B Infinite product measures and convolutions}

In this section we recall the definition and properties for infinite product measures and infinite convolution for complex Borel measures on $\mathbb{R}^{d}$. Denote by $\mathcal{B}\left(\mathbb{R}^{d}\right)^{\mathbb{N}}$ the 
On unique solutions to martingale problems

$\sigma$-algebra on $\left(\mathbb{R}^{d}\right)^{\mathbb{N}}$ which is generated by the mappings

$$
\pi_{n}:\left(\mathbb{R}^{d}\right)^{\mathbb{N}} \rightarrow \mathbb{R}^{d}, \quad\left(x_{k}\right)_{k \in \mathbb{N}} \mapsto x_{n} .
$$

It is also generated by the algebra

$$
\mathcal{R}:=\bigcup_{n \in \mathbb{N}} \sigma\left(\pi_{1}, \ldots, \pi_{n}\right) .
$$

Definition B.1. Let $\left(\mu_{n}\right)_{n \in \mathbb{N}}$ be a sequence of complex Borel measures on $\mathbb{R}^{d}$ and define $a_{n}:=\mu_{n}\left(\mathbb{R}^{d}\right), n \in \mathbb{N}$. Assume that $a:=\prod_{n=1}^{\infty} a_{n}$ exists in $\mathbb{C}$. A complex Borel measure $\mu$ on $\left(\left(\mathbb{R}^{d}\right)^{\mathbb{N}}, \mathcal{B}\left(\mathbb{R}^{d}\right)^{\mathbb{N}}\right)$ is the infinite product measure of $\left(\mu_{n}\right)_{n \in \mathbb{N}}$ if

$$
\int f\left(\pi_{1}, \ldots, \pi_{n}\right) d \mu=\frac{a}{\Pi_{j=1}^{n} a_{j}} \int \cdots \int f\left(x_{1}, \ldots, x_{n}\right) \mu_{1}\left(d x_{1}\right), \ldots, \mu_{n}\left(d x_{n}\right)
$$

for any $n \in \mathbb{N}$ and any bounded measurable function $f:\left(\mathbb{R}^{d}\right)^{n} \rightarrow \mathbb{R}$.

If it exists, the infinite product measure is unique because it is unique on $\mathcal{R}$. It is denoted by $\otimes_{n=1}^{\infty} \mu_{n}$. The following existence statement is classical.

Proposition B.2. Let $\left(P_{n}\right)_{n \in \mathbb{N}}$ be a sequence of probability measures on $\mathbb{R}^{d}$. Then there is a unique probability measure $P$ on $\left(\left(\mathbb{R}^{d}\right)^{\mathbb{N}}, \mathcal{B}\left(\mathbb{R}^{d}\right)^{\mathbb{N}}\right)$ such that $\left(\pi_{n}\right)_{n \in \mathbb{N}}$ is a sequence of independent random variables with $P^{\pi_{n}}=P_{n}$.

This can be easily lifted to finite measures as long as the product of their total mass converges to a finite non-zero number.

Lemma B.3. Let $\left(\mu_{n}\right)_{n \in \mathbb{N}}$ be a sequence of finite Borel measures on $\mathbb{R}^{d}$ and define $a_{n}:=\mu_{n}\left(\mathbb{R}^{d}\right)$ for any $n \in \mathbb{N}$. Assume that $a:=\prod_{n=1}^{\infty} a_{n} \in(0, \infty)$. Then the infinite product measure of $\left(\mu_{n}\right)_{n \in \mathbb{N}}$ exists.

Proof. Define $P_{n}:=\mu_{n} / a_{n}$. Then $\left(P_{n}\right)_{n \in \mathbb{N}}$ satisfies the requirements of Proposition B.2 and, hence, there is a probability measure $P$ as in Proposition B.2. The measure $\mu:=a P$ has the required property.

Proposition B.4. Let $\left(\mu_{n}\right)_{n \in \mathbb{N}}$ be a sequence of complex Borel measures on $\mathbb{R}^{d}$ and define $a_{n}:=\mu_{n}\left(\mathbb{R}^{d}\right)$ and $c_{n}:=\left|\mu_{n}\right|\left(\mathbb{R}^{d}\right)$ for any $n \in \mathbb{N}$. Assume that $c:=\Pi_{n=1}^{\infty} c_{n} \in(0, \infty)$ and that $a:=\prod_{n=1}^{\infty} a_{n}$ exists in $\mathbb{C}$. Then the infinite product measure $\left(\mu_{n}\right)_{n \in \mathbb{N}}$ exists and we have $\left|\otimes_{n=1}^{\infty} \mu_{n}\right|=\otimes_{n=1}^{\infty}\left|\mu_{n}\right|$.

Proof. By Lemma B.3 the infinite product measure $\nu$ of $\left(\left|\mu_{n}\right|\right)_{n \in \mathbb{N}}$ exists. Define a mapping $\tilde{\mu}: \mathcal{R} \rightarrow \mathbb{C}$ via

$$
\tilde{\mu}\left(\left(\pi_{1}, \ldots, \pi_{n}\right)^{-1}(A)\right)=\frac{a}{\Pi_{j=1}^{n} a_{j}} \int \ldots \int 1_{A}\left(x_{1}, \ldots, x_{n}\right) \mu_{1}\left(d x_{1}\right), \ldots, \mu_{n}\left(d x_{n}\right)
$$

for $A \in \mathcal{B}\left(\mathbb{R}^{d}\right)^{n}, n \in \mathbb{N}$. It is easy to verify that $\tilde{\mu}$ is a well-defined finitely additive measure on $\mathcal{R}$. Since $\nu$ is $\sigma$-additive and hence continuous in $\varnothing$, this also holds for $\tilde{\mu}$. Carathéodory's extension theorem yields that $\tilde{\mu}$ can be extended to a measure $\mu$ on $\mathcal{B}\left(\mathbb{R}^{d}\right)^{\mathbb{N}}$. Equation (B.2) implies that (B.1) holds and hence $\mu$ is the product measure of $\left(\mu_{n}\right)_{n \in \mathbb{N}}$. From Lemma A.1(11) it follows that $|\mu|$ coincides with $\otimes_{n=1}^{\infty}\left|\mu_{n}\right|$ on $\mathcal{R}$ and hence on $\mathcal{B}\left(\mathbb{R}^{d}\right)^{\mathbb{N}}=\sigma(\mathcal{R})$.

Now we turn to infinite convolutions which roughly coincide with pushforward measures of infinite sums of independent random variables. 
Definition B.5. Let $\left(\mu_{n}\right)_{n \in \mathbb{N}}$ be a sequence of complex Borel measures on $\mathbb{R}^{d}$. A complex Borel measure $\eta$ on $\mathbb{R}^{d}$ is the infinite convolution of $\left(\mu_{n}\right)_{n \in \mathbb{N}}$ if

$$
\int f(x) \eta(d x)=\lim _{n \rightarrow \infty} \int f(x)\left(*_{k=1}^{n} \mu_{k}\right)(d x)
$$

for any bounded continuous $f: \mathbb{R}^{d} \rightarrow \mathbb{R}$.

Observe that the infinite convolution is uniquely defined by the limiting property if it exists. It is denoted as $*_{n=1}^{\infty} \mu_{n}$. We give a simple criterion for its existence.

Proposition B.6. Let $\left(\mu_{n}\right)_{n \in \mathbb{N}}$ be a sequence of complex Borel measures on $\mathbb{R}^{d}$ and define $c_{n}:=\left|\mu_{n}\right|\left(\mathbb{R}^{d}\right)$ and $a_{n}:=\mu_{n}\left(\mathbb{R}^{d}\right)$ for any $n \in \mathbb{N}$. Assume that $c:=\Pi_{n=1}^{\infty} c_{n} \in$ $(0, \infty)$, that $a:=\Pi_{n=1}^{\infty} a_{n} \in \mathbb{C}$, that $\sum_{n=1}^{\infty} \int|v|^{2}\left|\mu_{n}\right|(d v)<\infty$, and that $\int v\left|\mu_{n}\right|(d v)=0$, $n \in \mathbb{N}$. Then the infinite convolutions of $\left(\mu_{n}\right)_{n \in \mathbb{N}}$ as well as $\left(\left|\mu_{n}\right|\right)_{n \in \mathbb{N}}$ exist. Moreover, $\left|*_{n=1}^{\infty} \mu_{n}\right| \leq *_{n=1}^{\infty}\left|\mu_{n}\right|$.

Proof. The measure $P:=\left|\otimes_{n=1}^{\infty} \mu_{n}\right| / c$ is a probability measure and $\pi_{n}$ is a sequence of independent random variables relative to $P$. Since $\lim _{n \rightarrow \infty} c_{n}=1$, we have

$$
\sum_{n=1}^{\infty} \int\left|\pi_{n}\right|^{2} d P=\sum_{n=1}^{\infty} c_{n}^{-1} \int|v|^{2}\left|\mu_{n}\right|(d v)<\infty
$$

and hence $\left(\sum_{k=1}^{n} \pi_{k}\right)_{n \in \mathbb{N}}$ converges in $L^{2}\left(\left(\mathbb{R}^{d}\right)^{\mathbb{N}}, \mathcal{B}\left(\mathbb{R}^{d}\right)^{\mathbb{N}}, P\right)$ and hence in probability to some random variable $S$. Set $\eta:=\left(\otimes_{n=1}^{\infty} \mu_{n}\right)^{S}$. For any bounded continuous function $f: \mathbb{R}^{d} \rightarrow \mathbb{R}$ we obtain

$$
\begin{aligned}
\left|\int\left(f(S)-f\left(\sum_{k=1}^{n} \pi_{k}\right)\right) d\left(\otimes_{n=1}^{\infty} \mu_{n}\right)\right| & \leq \int\left|f(S)-f\left(\sum_{k=1}^{n} \pi_{k}\right)\right| d\left|\otimes_{n=1}^{\infty} \mu_{n}\right| \\
& =c \int\left|f(S)-f\left(\sum_{k=1}^{n} \pi_{k}\right)\right| d P \\
& \rightarrow 0
\end{aligned}
$$

for $n \rightarrow \infty$ and hence

$$
\begin{aligned}
\int f(x) \eta(d x) & =\int f(S) d\left(\otimes_{n=1}^{\infty} \mu_{n}\right) \\
& =\lim _{n \rightarrow \infty} \int f\left(\sum_{k=1}^{n} \pi_{k}\right) d\left(\otimes_{n=1}^{\infty} \mu_{n}\right) \\
& =\lim _{n \rightarrow \infty} \frac{a}{\prod_{k=1}^{n} a_{k}} \int \cdots \int f\left(\sum_{k=1}^{n} x_{k}\right) \mu_{1}\left(d x_{1}\right) \cdots \mu_{n}\left(d x_{n}\right) \\
& =\lim _{n \rightarrow \infty} \int f(x)\left(*_{k=1}^{n} \mu_{k}\right)(d x)
\end{aligned}
$$

as desired for the infinite convolution. The existence of $*_{n=1}^{\infty}\left|\mu_{n}\right|$ follows along the same lines by setting $\gamma:=\left|\otimes_{n=1}^{\infty} \mu_{n}\right|^{S}$.

The last statement follows from $\left|\left(\otimes_{n=1}^{\infty} \mu_{n}\right)^{S}\right| \leq\left|\otimes_{n=1}^{\infty} \mu_{n}\right|^{S}$.

\section{References}

[1] V. Bogachev, P. Lescot, and M. Röckner. The martingale problem for pseudo-differential operators on infinite-dimensional spaces. Nogaya Mathematical Journal, 153:101-118, 1999. MR-1684553 
On unique solutions to martingale problems

[2] B. Böttcher. A parametrix construction for the fundamental solution of the evolution equation associated with a pseudo-differential operator generating a Markov process. Mathematische Nachrichten, 278:1235-1241, 2005. MR-2163294

[3] B. Böttcher, R. Schilling, and J. Wang. Lévy matters III. Lévy-Type Processes: Construction, Approximation and Sample Path Properties. Lecture Notes in Mathematics 2099. Springer, Berlin, 2013. MR-3156646

[4] C. Cuchiero. Affine and Polynomial Processes. PhD thesis, ETH Zürich, 2011.

[5] C. Cuchiero, M. Keller-Ressel, and J. Teichmann. Polynomial processes and their applications to mathematical finance. Finance and Stochastics, 16:711-740, 2012. MR-2972239

[6] N. Dinculeanu. Vector Measures. VEB Deutscher Verlag der Wissenschaft, Berlin, 1967. MR-0206189

[7] D. Duffie, D. Filipovic, and W. Schachermayer. Affine processes and applications in finance. The Annals of Applied Probability, 13:984-1053, 2003. MR-1994043

[8] E. Eberlein and J. Kallsen . Mathematical Finance. Springer, Cham, 2019. MR-3971545

[9] S. Ethier and T. Kurtz. Markov Processes. Characterization and Convergence. Wiley, New York, 1986. MR-0838085

[10] D. Filipović, and M. Larsson. Polynomial diffusions and applications in finance. Finance and Stochastics, 20:931-972, 2016. MR-3551857

[11] D. Filipović, and M. Larsson. Polynomial jump-diffusion models. arXiv preprint arXiv:1711.08043, 2017.

[12] L. Grafakos. Classical Fourier Analysis. Springer, New York, second edition, 2008. MR2445437

[13] H. Heuser. Lehrbuch der Analysis. Teil 1. Vieweg + Teubner, Wiesbaden, 1980. MR-0618121

[14] W. Hoh. Pseudo differential operators with negative definite symbols and the martingale problem. Stochastics and Stochastic Reports, 55:225-252, 1995. MR-1378858

[15] W. Hoh. A symbolic calculus for pseudo-differential operators generating Feller semigroups. Osaka Journal of Mathematics, 35:789-820, 1998. MR-1659620

[16] W. Hoh. Pseudo differential operators generating Markov processes. Habilitationsschrift, Universität Bielefeld, 1998.

[17] W. Hoh. Pseudo differential operators with negative definite symbols of variable order. Revista Matematica Iberoamericana, 16:219-241, 2000. MR-1809340

[18] N. Jacob. Further pseudodifferential operators generating Feller semigroups and Dirichlet forms. Revista Matematica Iberoamericana, 9:373-407, 1993. MR-1232848

[19] J. Jacod and P. Protter. Probability Essentials. Springer, Berlin, second edition, 2004. MR1956867

[20] N. Jacob and R. Schilling. Lévy-type processes and pseudodifferential operators. In O. Barndorff-Nielsen, T. Mikosch, and S. Resnick, editors, Lévy processes. Theory and Applications, pages 139-168. Birkhäuser, Boston, 2001. MR-1833696

[21] J. Jacod and A. Shiryaev. Limit Theorems for Stochastic Processes. Springer, Berlin, second edition, 2003. MR-1943877

[22] J. Kallsen. A didactic note on affine stochastic volatility models. In Yu. Kabanov, R. Liptser, and J. Stoyanov, editors, From Stochastic Calculus to Mathematical Finance, pages 343-368. Springer, Berlin, 2006. MR-2233549

[23] F. Kühn. On martingale problems and Feller processes. Electronic Journal of Probability, 23:1-18, 2018. MR-3771750

[24] F. Kühn. Existence of (Markovian) solutions to martingale problems associated with Lévy-type operators. Electronic Journal of Probability, 25:1-26, 2020. MR-4073677

[25] O. Okitaloshima and J. van Casteren. On the uniqueness of the martingale problem. International Journal of Mathematics, 7:775-810, 1996. MR-1417786

[26] T. Palmer. Banach Algebras and the General Theory of *-Algebras, volume I. Cambridge University Press, Cambridge, 1994. MR-1270014 
On unique solutions to martingale problems

[27] D. Revuz and M. Yor. Continuous Martingales and Brownian Motion. Springer, Berlin, third edition, 1999. MR-1725357

[28] C. Rogers and D. Williams. Diffusions, Markov processes and Martingales: Volume 2, Itō Calculus. Cambridge University Press, 2000. MR-1780932

[29] K. Sato. Lévy Processes and Infinitely Divisible Distributions. Cambridge University Press, Cambridge, 1999. MR-1739520

[30] D. Stroock. Diffusion processes associated with Lévy generators. Zeitschrift für Wahrscheinlichkeitstheorie und verwandte Gebiete, 32:209-244, 1975. MR-0433614

[31] E. Veerman. Affine Markov Processes on a General State Space. PhD thesis, University of Amsterdam, 2011. 


\section{Electronic Journal of Probability Electronic Communications in Probability}

\section{Advantages of publishing in EJP-ECP}

- Very high standards

- Free for authors, free for readers

- Quick publication (no backlog)

- Secure publication $\left(\mathrm{LOCKSS}^{1}\right)$

- Easy interface (EJMS²)

\section{Economical model of EJP-ECP}

- Non profit, sponsored by $\mathrm{IMS}^{3}, \mathrm{BS}^{4}$, ProjectEuclid ${ }^{5}$

- Purely electronic

\section{Help keep the journal free and vigorous}

- Donate to the IMS open access fund ${ }^{6}$ (click here to donate!)

- Submit your best articles to EJP-ECP

- Choose EJP-ECP over for-profit journals

\footnotetext{
${ }^{1}$ LOCKSS: Lots of Copies Keep Stuff Safe http://www. lockss.org/

${ }^{2}$ EJMS: Electronic Journal Management System http://www.vtex.lt/en/ejms.html

${ }^{3}$ IMS: Institute of Mathematical Statistics http://www.imstat.org/

${ }^{4}$ BS: Bernoulli Society http://www. bernoulli-society.org/

${ }^{5}$ Project Euclid: https://projecteuclid.org/

${ }^{6}$ IMS Open Access Fund: http://www.imstat.org/publications/open.htm
} 